\title{
TITLE:
}

\section{Open-closed homotopy algebra in mathematical physics}

$\operatorname{AUTHOR}(\mathrm{S}):$

Kajiura, H; Stasheff, J

\section{CITATION:}

Kajiura, $\mathrm{H}$... [et al]. Open-closed homotopy algebra in mathematical physics. JOURNAL OF MATHEMATICAL PHYSICS 2006, 47(2): 023506.

\section{ISSUE DATE:}

2006-02

URL:

http://hdl.handle.net/2433/50497

\section{RIGHT:}

Copyright 2006 American Institute of Physics. This article may be downloaded for personal use only. Any other use requires prior permission of the author and the American Institute of Physics. 


\title{
Open-closed homotopy algebra in mathematical physics
}

\author{
Hiroshige Kajiura ${ }^{\text {a) }}$ \\ Yukawa Institute for Theoretical Physics, Kyoto University, Kyoto 606-8502, Japan \\ Jim Stasheff ${ }^{\text {b) }}$ \\ Department of Mathematics, University of Pennsylvania, Philadelphia, \\ Pennsylvania 19104-6395
}

(Received 14 November 2005; accepted 10 January 2006;

published online 28 February 2006)

In this paper we discuss various aspects of open-closed homotopy algebras (OCHAs) presented in our previous paper, inspired by Zwiebach's open-closed string field theory, but that first paper concentrated on the mathematical aspects. Here we show how an OCHA is obtained by extracting the tree part of Zwiebach's quantum open-closed string field theory. We clarify the explicit relation of an OCHA with Kontsevich's deformation quantization and with the B-models of homological mirror symmetry. An explicit form of the minimal model for an OCHA is given as well as its relation to the perturbative expansion of open-closed string field theory. We show that our open-closed homotopy algebra gives us a general scheme for deformation of open string structures $\left(A_{\infty}\right.$ algebras $)$ by closed strings ( $L_{\infty}$ algebras). (C) 2006 American Institute of Physics. [DOI: 10.1063/1.2171524]

\section{INTRODUCTION}

In this paper we discuss various aspects of open-closed homotopy algebras (OCHAs) defined in our previous paper. ${ }^{35}$ They are a kind of homotopy algebra inspired by Zwiebach's classical open-closed string field theory ${ }^{74}$ and also related to the deformation quantization setup by Kontsevich. ${ }^{40}$ In Ref. 35 we showed that an OCHA actually defines a homotopy invariant algebraic structure and also it gives us a general scheme for deformation of open string structures $\left(A_{\infty}\right.$ algebras) by closed strings ( $L_{\infty}$ algebras).

As tree closed strings and open strings are related to the conformal plane $\mathrm{C}$ with punctures and the upper half plane $H$ with punctures on the boundary, respectively, tree open-closed strings are related to the upper half plane $H$ with punctures both in the bulk and on the boundary, which appears recently in the context of deformation quantization. ${ }^{40}$ In operad theory (see Ref. 53), the relevance of the little disk operad to closed string theory is known. The little interval operad and associahedra are relevant to open string theory. The Swiss-cheese operad, ${ }^{69}$ that combines the little disk operad with the little interval operad, also is inspired by Kontsevich's approach to deformation quantization. Our OCHA should be homotopy equivalent to a part of an algebra over the Swiss-cheese operad. It should be very interesting to investigate the remaining structures (see Ref. 23 , which is related to this direction).

We first present the definition of OCHAs together with recalling two typical homotopy algebras, $A_{\infty}$ algebras and $L_{\infty}$ algebras, in Sec. II.

In Sec. III, we give an alternate interpretation in terms of odd formal vector fields (often called homological vector fields) on a supermanifold, which we believe is a more acceptable description for physicists.

\footnotetext{
${ }^{a)}$ Electronic mail: kajiura@yukawa.kyoto-u.ac.jp

${ }^{b)}$ Electronic mail: jds@math.upenn.edu
} 
The connection to classical open-closed string field theory by Zwiebach ${ }^{74}$ is given in Sec. IV. It is known that classical closed string field theory has an $L_{\infty}$ structure, ${ }^{73,66,38}$ and classical open string field theory has an $A_{\infty}$ structure. ${ }^{11,74,55,31}$ We show that an OCHA is obtained by extracting the tree part of Zwiebach's quantum open-closed string field theory. Since in general homotopy algebras are something whose structures are governed by the underlying tree graph (operad) structure, the structures of quantum string field theories are something beyond the ordinary homotopy algebra (see loop homotopy algebras ${ }^{50}$ for quantum closed string field theories). Thus, we can say that OCHAs are the maximal homotopy algebraic structures which string field theories should have. Namely,

\section{Quantum open-closed SFT $\supset$ OCHA $\supset L_{\infty} \oplus A_{\infty}$.}

One of the key theorems in homotopy algebra is the minimal model theorem which was proven for $A_{\infty}$ algebras by Kadeishvili. ${ }^{30}$ It holds true also for $L_{\infty}$ algebras in a similar way, and in our previous paper ${ }^{35}$ we stated the minimal model theorem holds for OCHAs, too. In Sec. V we present an explicit way of constructing a minimal model for an OCHA, and explain its relation to the perturbative expansion of classical open-closed string field theory.

Section VI is devoted to explaining some deformation theory aspect of OCHAs. An openclosed homotopy algebra consists of a direct sum of graded vector spaces $\mathcal{H}=\mathcal{H}_{c} \oplus \mathcal{H}_{o}$. It has an $L_{\infty}$ structure on $\mathcal{H}_{c}$ and reduces to an $A_{\infty}$ algebra if we set $\mathcal{H}_{c}=0$. From such a viewpoint, an open-closed homotopy algebra gives a general scheme of deformation of the $A_{\infty}$ algebra by $\mathcal{H}_{c}$, where the deformation space is parametrized by a moduli space of the $L_{\infty}$ algebra on $\mathcal{H}_{c}{ }^{35}$ In Sec. VI A we recall this fact in a more explicit way than Ref. 35. After that, we explain the relation of this viewpoint to various aspects of string theory; Kontsevich's deformation quantization ${ }^{40}$ in Sec. VI B, and open-closed B-models (cf. Ref. 26) in Sec. VI C.

\section{OPEN-CLOSED HOMOTOPY ALGEBRA}

An open-closed homotopy algebra, as we proposed in our previous paper, ${ }^{35}$ is a homotopy algebra which combines two typical homotopy algebras, an $A_{\infty}$ algebra and an $L_{\infty}$ algebra. There are various equivalent ways of defining and/or describing strong homotopy algebras. In this paper, we shall present them in terms of multivariable operations in this section, and in Sec. III we shall reinterpret them in terms of the supermanifold description. For the equivalent coalgebra description and tree graph description, see Ref. 35. Here we recall just enough so that this paper can be read without having to read Ref. 35. The reader familiar with $A_{\infty}$ algebras and $L_{\infty}$ algebras can go directly to Definition 2.8.

We first begin with recalling $A_{\infty}$ algebras and $L_{\infty}$ algebras in Sec. II A. The definition of open-closed homotopy algebras are given in Sec. II B. In Sec. II C we define cyclic structures in open-closed homotopy algebras together with explaining some background of such structures.

We restrict our arguments to the case that the characteristic of the field $k$ is zero. We further let $k=\mathrm{C}$ for simplicity.

\section{A. $A_{\infty}$ algebras and $L_{\infty}$ algebras}

Definition 2.1 [ $A_{\infty}$ algebra (strong homotopy associative algebra) $\left.{ }^{64}\right]$ : Let $\mathcal{H}_{o}$ be a Z-graded vector space $\mathcal{H}_{o}=\oplus_{r \in \mathbb{Z}} \mathcal{H}_{o}^{r}$ and suppose that there exists a collection of degree one multilinear maps

$$
\mathfrak{m}:=\left\{m_{k}:\left(\mathcal{H}_{o}\right)^{\otimes k} \rightarrow \mathcal{H}_{o}\right\}_{k \geqslant 1} .
$$

$\left(\mathcal{H}_{o}, \mathfrak{m}\right)$ is called an $A_{\infty}$ algebra when the multilinear maps $m_{k}$ satisfy the following relations:

$$
\sum_{k+l=n+1} \sum_{j=0}^{k-1}(-1)^{o_{1}+\cdots+o_{j}} m_{k}\left(o_{1}, \ldots, o_{j}, m_{l}\left(o_{j+1}, \ldots, o_{j+l}\right), o_{j+l+1}, \ldots, o_{n}\right)=0
$$

for $n \geqslant 1$, where $o_{i}$ on $(-1)$ denotes the degree of $o_{i}$. A weak $A_{\infty}$ algebra $\left(\mathcal{H}_{o}, \mathfrak{m}\right)$ consists of a 
collection of degree one multilinear maps $\mathfrak{m}:=\left\{m_{k}:\left(\mathcal{H}_{o}\right)^{\otimes k} \rightarrow \mathcal{H}_{o}\right\}_{k \geqslant 0}$ satisfying the corresponding relations

$$
\sum_{k+l=n+1} \sum_{j=0}^{k-1}(-1)^{o_{1}+\cdots+o_{j}} m_{k}\left(o_{1}, \ldots, o_{j}, m_{l}\left(o_{j+1}, \ldots, o_{j+l}\right), o_{j+l+1}, \ldots, o_{n}\right)=0
$$

for $n \geqslant 0$.

Remark 2.2: The definition above is different from the original one ${ }^{64}$ in the definition of the degree of the multilinear maps $m_{k}$. Both are in fact equivalent and related by suspension. ${ }^{15,53}$ In Ref. 64, the $m_{k}$ are multilinear maps on $\downarrow \mathcal{H}_{o}$ where $\left(\downarrow \mathcal{H}_{o}\right)^{r+1}=\mathcal{H}_{o}^{r}$; in algebraic topology the desuspension is denoted by $\downarrow$, which is equivalent to $[-1]$ in the algebraic geometry tradition: $\downarrow \mathcal{H}_{o}=\mathcal{H}_{o}[-1]$. Since it might be more familiar also in mathematical physics as in Sec. VI, in this paper we denote the suspension and desuspension by [1] and [-1], respectively.

For an $A_{\infty}$ algebra $\left(\mathcal{H}_{o}, \mathfrak{m}\right)$ (in the case $\left.m_{0}=0\right)$, the first three relations of the $A_{\infty}$ condition (2.1) are

$$
\begin{gathered}
0=m_{1}^{2}, \\
0=m_{1}\left(m_{2}\left(o_{1}, o_{2}\right)\right)+m_{2}\left(m_{1}\left(o_{1}\right), o_{2}\right)+(-1)^{o_{1}} m_{2}\left(o_{1}, m_{1}\left(o_{2}\right)\right), \\
0=m_{1}\left(m_{3}\left(o_{1}, o_{2}, o_{3}\right)\right)+m_{3}\left(m_{1}\left(o_{1}\right), o_{2}, o_{3}\right)+(-1)^{o_{1}} m_{3}\left(o_{1}, m_{1}\left(o_{2}\right), o_{3}\right)+(-1)^{o_{1}+o_{2}} m_{3}\left(o_{1}, o_{2}, m_{1}\left(o_{3}\right)\right) \\
+m_{2}\left(m_{2}\left(o_{1}, o_{2}\right), o_{3}\right)+(-1)^{o_{1}} m_{2}\left(o_{1}, m_{2}\left(o_{2}, o_{3}\right)\right) .
\end{gathered}
$$

The first equation, in the physics terminology, says $m_{1}$ is nilpotent; $\left(\mathcal{H}_{o}, m_{1}\right)$ defines a complex on the Z-graded vector space $\mathcal{H}_{o}$. The second equation says the differential $m_{1}$ satisfies the Leibniz rule for the product $m_{2}$. The third equation means the product $m_{2}$ is associative up to the term including $m_{3}$. Thus, a differential graded algebra (DGA) is described as an $A_{\infty}$ algebra on $\downarrow \mathcal{H}_{o}$ $=\mathcal{H}_{o}[-1]$ with a differential $m_{1}$, a product $m_{2}$, and $m_{3}=m_{4}=\cdots=0$.

Definition 2.3 ( $A_{\infty}$ morphism): For two $A_{\infty}$ algebras $\left(\mathcal{H}_{o}, \mathfrak{m}\right)$ and $\left(\mathcal{H}_{o}^{\prime}, \mathfrak{m}^{\prime}\right)$, suppose that there exists a collection of degree zero (degree preserving) multilinear maps

$$
f_{k}: \mathcal{H}_{o}^{\otimes k} \rightarrow \mathcal{H}_{o}^{\prime}, \quad k \geqslant 1 .
$$

The collection $\left\{f_{k}\right\}_{k \geqslant 1}:\left(\mathcal{H}_{o}, \mathfrak{m}\right) \rightarrow\left(\mathcal{H}_{o}^{\prime}, \mathfrak{m}^{\prime}\right)$ is called an $A_{\infty}$ morphism iff it satisfies the following relations:

$$
\begin{aligned}
& \sum_{1 \leqslant k_{1}<k_{2} \ldots<k_{i}=n} m_{i}^{\prime}\left(f_{k_{1}}\left(o_{1}, \ldots, o_{k_{1}}\right), f_{k_{2}-k_{1}}\left(o_{k_{1}+1}, \ldots, o_{k_{2}}\right) \cdots f_{n-k_{i-1}}\left(o_{k_{i-1}+1}, \ldots, o_{n}\right)\right) \\
= & \sum_{k+l=n+1} \sum_{j=0}^{k-1}(-1)^{o_{1}+\cdots+o_{j}} f_{k}\left(o_{1}, \ldots, o_{j}, m_{l}\left(o_{j+1}, \ldots, o_{j+l}\right), o_{j+l+1}, \ldots, o_{n}\right)
\end{aligned}
$$

for $n \geqslant 1$. If $\left(\mathcal{H}_{o}, \mathfrak{m}\right)$ and $\left(\mathcal{H}_{o}^{\prime}, \mathfrak{m}^{\prime}\right)$ are weak $A_{\infty}$ algebras, then a weak $A_{\infty}$ morphism consists of multilinear maps $\left\{f_{k}\right\}_{k \geqslant 0}$, where $f_{0}: \mathrm{C} \rightarrow A^{\prime}$, satisfying the above conditions and in addition

$$
f_{1} \circ m_{0}=\sum m_{k}^{\prime}\left(f_{0}, \ldots, f_{0}\right) .
$$

As an $A_{\infty}$ algebra can be thought of as a generalization of a differential graded algebra (DGA), an $L_{\infty}$ algebra is a generalization of a differential graded Lie algebra (DGLA). As ordinary associative and Lie algebras are related by skew-symmetrization and the universal enveloping construction, there are corresponding relations for $A_{\infty}$ algebras and $L_{\infty}$ algebras. ${ }^{42}$

Definition 2.4 (graded symmetry): A graded symmetric multilinear map of a graded vector 
space $V$ to itself is a linear map $f: V^{\otimes n} \rightarrow V$ such that, for any $c_{i} \in V 1 \leqslant i \leqslant n$ and any $\sigma \in \mathfrak{S}_{n}$ (the permutation group of $n$ elements), the relation

$$
f\left(c_{1}, \ldots, c_{n}\right)=(-1)^{\epsilon(\sigma)} f\left(c_{\sigma(1)}, \ldots, c_{\sigma(n)}\right)
$$

holds, where the sign $(-1)^{\epsilon(\sigma)}$ is the Koszul sign of the permutation $\sigma$.

Also we adopt the convention that tensor products of functions or operators have the signs built in; e.g., $(f \otimes g)(x \otimes y)=(-1)^{g \cdot x} f(x) \otimes g(y)$.

Definition $2.5\left[L_{\infty}\right.$ algebra (strong homotopy Lie algebra $\left.{ }^{43}\right]$ : Let $\mathcal{H}_{c}$ be a graded vector space and suppose that a collection of degree one graded symmetric multilinear maps $\mathfrak{l}:=\left\{l_{k}: \mathcal{H}_{c}^{\otimes k}\right.$ $\left.\rightarrow \mathcal{H}_{c}\right\}_{l \geqslant 0}$ is given. $\left(\mathcal{H}_{c}, \mathfrak{l}\right)$ is called a weak $L_{\infty}$ algebra iff the multilinear maps satisfy the following relations:

$$
\sum_{k+l=n+1} \sum_{\sigma \in \mathfrak{S}_{n}} \frac{(-1)^{\epsilon(\sigma)}}{l !(n-l) !} l_{k}\left(l_{l}\left(c_{\sigma(1)}, \ldots, c_{\sigma(l)}\right), c_{\sigma(l+1)}, \ldots, c_{\sigma(n)}\right)=0
$$

for $n \geqslant 0$. If the relation is satisfied for $n \geqslant 1$ without the additional map $l_{0}: C_{\rightarrow} \rightarrow \mathcal{H}_{c}^{1} \subset \mathcal{H}_{c}$, then $\left(\mathcal{H}_{c}, \mathfrak{l}\right)$ is called an $L_{\infty}$ algebra.

Remark 2.6: $L_{\infty}$ algebras are usually defined in a similar but different fashion, where the summation for the permutation $\mathfrak{S}_{n}$ in Eq. (2.4) is replaced by the summation over the unshuffle permutations (2.3). This unshuffled description would enable us to drop all the symmetrization factors in this paper. However, we take the one with all the permutations since it fits the dual description in the next section.

For an $L_{\infty}$ algebra $\left(\mathcal{H}_{c}, \mathfrak{l}\right)$, the first three relations of the $L_{\infty}$ condition (2.4) are

$$
\begin{gathered}
0=\left(l_{1}\right)^{2}, \\
0=l_{1}\left(l_{2}\left(c_{1}, c_{2}\right)\right)+l_{2}\left(l_{1}\left(c_{1}\right), c_{2}\right)+(-1)^{c_{1}} l_{2}\left(c_{1}, l_{1}\left(c_{2}\right)\right), \\
0=l_{1}\left(l_{3}\left(c_{1}, c_{2}, c_{3}\right)\right)+l_{3}\left(l_{1}\left(c_{1}\right), c_{2}, c_{3}\right)+(-1)^{c_{1}} l_{3}\left(c_{1}, l_{1}\left(c_{2}\right), c_{3}\right)+(-1)^{c_{1}+c_{2}} l_{3}\left(c_{1}, c_{2}, l_{1}\left(c_{3}\right)\right) \\
+l_{2}\left(l_{2}\left(c_{1}, c_{2}\right), c_{3}\right)+(-1)^{c_{1}\left(c_{2}+c_{3}\right)} l_{2}\left(l_{2}\left(c_{2}, c_{3}\right), c_{1}\right)+(-1)^{c_{3}\left(c_{1}+c_{2}\right)} l_{2}\left(l_{2}\left(c_{3}, c_{1}\right), c_{2}\right) .
\end{gathered}
$$

As in the case of an $A_{\infty}$ algebra, the first equation indicates that $\left(\mathcal{H}_{c}, l_{1}\right)$ defines a complex, while, after a shift in grading, the second equation implies the differential $l_{1}$ satisfies a Leibniz rule with respect to the Lie bracket $l_{2}$, and the third equation means the bracket $l_{2}$ satisfies the Jacobi identity up to the terms including $l_{3}$. Thus, a differential graded Lie algebra is described as an $L_{\infty}$ algebra on $\downarrow \mathcal{H}_{c}=\mathcal{H}_{c}[-1]$ with a differential $l_{1}$, a Lie bracket $l_{2}$, and $l_{3}=l_{4}=\cdots=0$.

Definition $2.7\left(L_{\infty}\right.$ morphism): For two weak $L_{\infty}$ algebras $\left(\mathcal{H}_{c}, \mathfrak{l}\right)$ and $\left(\mathcal{H}_{c}^{\prime}, \mathfrak{l}^{\prime}\right)$, suppose that there exists a collection of degree zero (degree preserving) graded symmetric multilinear maps

$$
f_{k}: \mathcal{H}_{c}^{\otimes k} \rightarrow \mathcal{H}_{c}^{\prime}, \quad l \geqslant 0 .
$$

Here $f_{0}$ is a map from $\mathrm{C}$ to a degree zero subvector space of $\mathcal{H}_{c}$. The collection $\left\{f_{k}\right\}_{k \geqslant 0}:\left(\mathcal{H}_{c}, \mathfrak{l}\right)$ $\rightarrow\left(\mathcal{H}_{c}^{\prime}, \mathfrak{l}^{\prime}\right)$ is called a weak $L_{\infty}$ morphism iff it satisfies the following relations:

$$
\begin{aligned}
& \sum_{k+l=n+1} \sum_{\sigma \in \mathfrak{S}_{n}} \frac{(-1)^{\epsilon(\sigma)}}{l !(n-l) !} f_{k}\left(l_{l} \otimes \mathbf{1}_{c}^{\otimes n-l}\right)\left(c_{\sigma(1)}, \ldots, c_{\sigma(n)}\right)=\sum_{k_{1}+\cdots+k_{j}=n} \sum_{\sigma \in \mathfrak{S}_{n}} \frac{(-1)^{\epsilon(\sigma)}}{k_{1} ! k_{2} ! \cdots k_{j} ! \cdot j !} l_{j}^{\prime}\left(f_{k_{1}} \otimes f_{k_{2}}\right. \\
& \left.\otimes \cdots \otimes f_{k_{j}}\right)\left(c_{\sigma(1)}, \ldots, c_{\sigma(n)}\right)
\end{aligned}
$$

for $n \geqslant 0$. In particular, when $\left(\mathcal{H}_{c}, \mathfrak{l}\right)$ and $\left(\mathcal{H}_{c}^{\prime}, \mathfrak{l}^{\prime}\right)$ are $L_{\infty}$ algebras, a weak $L_{\infty}$ morphism $\left\{f_{k}\right\}_{k \geqslant 0}:\left(\mathcal{H}_{c}, \mathfrak{l}\right) \rightarrow\left(\mathcal{H}_{c}^{\prime}, \mathfrak{l}^{\prime}\right)$ is called an $L_{\infty}$ morphism if in addition $f_{0}=0$. 


\section{B. Open-closed homotopy algebra}

Definition 2.8 (open-closed homotopy algebra ${ }^{35}$ ): Let $\mathcal{H}=\mathcal{H}_{c} \oplus \mathcal{H}_{o}$ be a graded vector space and $\left(\mathcal{H}_{c}, l\right)$ be a weak $L_{\infty}$ algebra. Consider a collection of multilinear maps

$$
\mathfrak{n}:=\left\{n_{k, l}:\left(\mathcal{H}_{c}\right)^{\otimes k} \otimes\left(\mathcal{H}_{o}\right)^{\otimes l} \rightarrow \mathcal{H}_{o}\right\}_{k, l \geqslant 0}
$$

each of which is graded symmetric on $\left(\mathcal{H}_{c}\right)^{\otimes l}$. We call $(\mathcal{H}, \mathfrak{l}, \mathfrak{n})$ a weak open-closed homotopy algebra (weak OCHA) when $\mathfrak{n}$ satisfies the following relations:

$$
\begin{aligned}
0= & \sum_{p+r=n} \sum_{\sigma \in \mathfrak{S}_{n}} \frac{(-1)^{\epsilon(\sigma)}}{p ! r !} n_{1+r, m}\left(l_{p}\left(c_{\sigma(1)}, \ldots, c_{\sigma(p)}\right), c_{\sigma(p+1)}, \ldots, c_{\sigma(n)} ; o_{1}, \ldots, o_{m}\right) \\
& +\sum_{p+r=n} \sum_{i+s+j=m} \sum_{\sigma \in \mathfrak{S}_{n}} \frac{(-1)^{\mu_{p, i}(\sigma)}}{p ! r !} \\
& \times n_{p, i+1+j}\left(c_{\sigma(1)}, \ldots, c_{\sigma(p)} ; o_{1}, \ldots, o_{i}, n_{r, s}\left(c_{\sigma(p+1)}, \ldots, c_{\sigma(n)} ; o_{i+1}, \ldots, o_{i+s}\right), o_{i+s+1}, \ldots, o_{m}\right) .
\end{aligned}
$$

Here the sign exponent $\mu_{p, i}(\sigma)$ is given explicitly by

$$
\mu_{p, i}(\sigma)=\epsilon(\sigma)+\left(c_{\sigma(1)}+\cdots+c_{\sigma(p)}\right)+\left(o_{1}+\cdots+o_{i}\right)+\left(o_{1}+\cdots+o_{i}\right)\left(c_{\sigma(p+1)}+\cdots+c_{\sigma(n)}\right),
$$

corresponding to the signs effected by the interchanges. In particular, if $l_{0}=n_{0,0}=0$, we call $(\mathcal{H}, \mathfrak{l}, \mathfrak{n})$ an open-closed homotopy algebra. We can also write the defining equation (2.6) in the following shorthand expression:

$$
\begin{gathered}
0=\sum_{p+r=n} \sum_{\sigma \in \mathfrak{S}_{n}} \frac{(-1)^{\epsilon(\sigma)}}{p ! r !}(-1)^{\epsilon(\sigma)} n_{1+r, m}\left(\left(l_{p} \otimes \mathbf{1}_{c}^{\otimes r} \otimes \mathbf{1}_{o}^{\otimes m}\right)\left(c_{\sigma(1)}, \ldots, c_{\sigma(n)} ; o_{1}, \ldots, o_{m}\right)\right) \\
+\sum_{p+r=n} \sum_{i+s+j=m} \sum_{\sigma \in \mathfrak{S}_{n}} \frac{(-1)^{\epsilon(\sigma)}}{p ! r !} n_{p, i+1+j}\left(\left(\mathbf{1}_{c}^{\otimes p} \otimes \mathbf{1}_{o}^{\otimes i} \otimes n_{r, s} \otimes \mathbf{1}_{o}^{\otimes j}\right)\left(c_{\sigma(1)}, \ldots, c_{\sigma(n)} ; o_{1}, \ldots, o_{m}\right)\right),
\end{gathered}
$$

where the complicated sign is absorbed into this expression.

Remark 2.9: For an OCHA $(\mathcal{H}, \mathfrak{l}, \mathfrak{n})$, the substructure $\left(\mathcal{H}_{c}, \mathfrak{l}\right)$ is by definition an $L_{\infty}$ algebra and $\left(\mathcal{H}_{o},\left\{n_{0, k}\right\}\right)$ forms an $A_{\infty}$ algebra. Furthermore, the substructure $\left(\mathcal{H},\left\{n_{1, q}\right\}_{q \geqslant 0}\right)$ forms an $A_{\infty}$ module over the $A_{\infty}$ algebra $\left(\mathcal{H}_{o}, \mathfrak{m}\right)$ in the sense of Refs. 49 and 67. Also, if $n_{p, 0}=0$ for all $p \geqslant 1$, the substructure $\left(\mathcal{H},\left\{n_{p, 1}\right\}\right)$ makes $\mathcal{H}_{o}$ an $L_{\infty}$ module over $\left(\mathcal{H}_{c}, \mathfrak{l}\right) .{ }^{42}$

Now, let us denote $l_{1}=d_{c}$ and $n_{0,1}=d_{o}$. The first few relations which do not appear as $A_{\infty}$ or $L_{\infty}$ conditions are

$$
\begin{gathered}
0=d_{o} n_{1,0}+n_{1,0} d_{c}, \\
0=d_{o} n_{1,1}(c ; o)+n_{1,1}\left(c ; d_{o}(o)\right)+n_{1,1}\left(d_{c}(c) ; o\right)+n_{0,2}\left(n_{1,0}(c), o\right)+(-1)^{c(o+1)} n_{0,2}\left(o, n_{1,0}(c)\right),
\end{gathered}
$$

$$
\begin{aligned}
0= & d_{o} n_{2,0}\left(c_{1}, c_{2}\right)+n_{2,0}\left(d_{c}\left(c_{1}\right), c_{2}\right)+(-1)^{c_{1} c_{2}} n_{2,0}\left(d_{c}\left(c_{2}\right), c_{1}\right)+n_{1,0} l_{2}\left(c_{1}, c_{2}\right)+(-1)^{c_{1}} n_{1,1}\left(c_{1}, n_{1,0}\left(c_{2}\right)\right) \\
& +(-1)^{c_{2}\left(1+c_{1}\right)} n_{1,1}\left(c_{2}, n_{1,0}\left(c_{1}\right)\right),
\end{aligned}
$$




$$
\begin{aligned}
0= & d_{o} n_{1,2}\left(c ; o_{1}, o_{2}\right)+n_{1,2}\left(d_{c}(c) ; o_{1}, o_{2}\right)+(-1)^{c} n_{1,2}\left(c ; d_{o}\left(o_{1}\right), o_{2}\right)+(-1)^{c+o_{1}} n_{1,2}\left(c ; o_{1}, d_{o}\left(o_{2}\right)\right) \\
+ & n_{1,1}\left(c, n_{0,2}\left(o_{1}, o_{2}\right)\right)+n_{0,2}\left(n_{1,1}\left(c ; o_{1}\right), o_{2}\right)+(-1)^{o_{1}(1+c)} n_{0,2}\left(o_{1}, n_{1,1}\left(c, o_{2}\right)\right)+n_{0,3}\left(n_{1,0}(c), o_{1}, o_{2}\right) \\
+ & (-1)^{o_{1}(1+c)} n_{0,3}\left(o_{1}, n_{1,0}(c), o_{2}\right)+(-1)^{\left(o_{1}+o_{2}\right)(1+c)} n_{0,3}\left(o_{1}, o_{2}, n_{1,0}(c)\right), \\
0= & d_{o} n_{2,1}\left(c_{1}, c_{2} ; o\right)+n_{2,1}\left(d_{c}\left(c_{1}\right), c_{2} ; o\right)+(-1)^{c_{1}} n_{2,1}\left(c_{1}, d_{c}\left(c_{2}\right) ; o\right)+(-1)^{c_{1}+c_{2}} n_{2,1}\left(c_{1}, c_{2} ; d_{o}(o)\right) \\
& +n_{1,1}\left(l_{2}\left(c_{1}, c_{2}\right) ; o\right)++(-1)^{c_{1}} n_{1,1}\left(c_{1} ; n_{1,1}\left(c_{2} ; o\right)\right)+(-1)^{c_{2}\left(c_{1}+1\right)} n_{1,1}\left(c_{2} ; n_{1,1}\left(c_{1} ; o\right)\right) \\
& +n_{0,2}\left(n_{2,0}\left(c_{1}, c_{2}\right), o\right)+(-1)^{o\left(1+c_{1}+c_{2}\right)} n_{0,2}\left(o, n_{2,0}\left(c_{1}, c_{2}\right)\right)+(-1)^{c_{2}\left(1+c_{1}\right)} n_{1,2}\left(c_{2} ; n_{1,0}\left(c_{1}\right), o\right) \\
& +(-1)^{\left(c_{2}+o\right)\left(1+c_{1}\right)} n_{1,2}\left(c_{2} ; o, n_{1,0}\left(c_{1}\right)\right)+(-1)^{c_{1}} n_{1,2}\left(c_{1} ; n_{1,0}\left(c_{2}\right), o\right) \\
& +(-1)^{c_{1}+o\left(1+c_{2}\right)} n_{1,2}\left(c_{1} ; o, n_{1,0}\left(c_{2}\right)\right), \ldots .
\end{aligned}
$$

Equation (2.8) implies $n_{1,0}$ is a chain map by an appropriate relative shift of the grading. On the other hand, in the case $n_{0,1}=0$, Eq. (2.9) is an extended Leibniz rule. Suppose that we have an OCHA with only nonzero structures $d_{c}, d_{o}, l_{2}, n_{1,1}, m_{2}:=n_{0,2}$. In Eq. (2.11) only the second line survives, which means that $\mathcal{H}_{c}$ acts on an algebra $\left(\mathcal{H}_{o}, m_{2}\right)$ by $n_{1,1}$ as derivations. Furthermore, in Eq. (2.12) only the second line survives, which implies that $\mathcal{H}_{o}$ represents a Lie algebra $\left(\mathcal{H}_{c}, l_{2}\right)$. Then $\left(\mathcal{H}, d_{c}, d_{o}, l_{2}, n_{1,1}, m_{2}\right)$ forms what is called a $\mathfrak{g}$ algebra or Leibniz pair (see Ref. 35 and references there).

Definition 2.10 (open-closed homotopy algebra morphism): For two weak OCHAs $(\mathcal{H}, \mathfrak{l}, \mathfrak{n}$ ) and $\left(\mathcal{H}^{\prime}, \mathfrak{l}^{\prime}, \mathfrak{n}^{\prime}\right)$, consider a collection $\mathfrak{f}$ of degree zero (degree preserving) multilinear maps

$$
\begin{gathered}
f_{k}:\left(\mathcal{H}_{c}\right)^{\otimes k} \rightarrow \mathcal{H}_{c}^{\prime} \quad \text { for } k \geqslant 0, \\
f_{k, l}:\left(\mathcal{H}_{c}\right)^{\otimes k} \otimes\left(\mathcal{H}_{o}\right)^{\otimes l} \rightarrow \mathcal{H}_{o}^{\prime} \quad \text { for } k, l \geqslant 0,
\end{gathered}
$$

where $f_{k}$ and $f_{k, l}$ are graded symmetric with respect to $\left(\mathcal{H}_{c}\right)^{\otimes k}$. We call $\mathfrak{f}:(\mathcal{H}, \mathfrak{l}, \mathfrak{n}) \rightarrow\left(\mathcal{H}^{\prime}, \mathfrak{l}^{\prime}, \mathfrak{n}^{\prime}\right)$ a weak OCHA morphism when $\left\{f_{k}\right\}_{k \geqslant 0}:\left(\mathcal{H}_{c}, \mathfrak{l}\right) \rightarrow\left(\mathcal{H}_{c}^{\prime}, l^{\prime}\right)$ is a weak $L_{\infty}$ morphism and $\left\{f_{k, l}\right\}_{k, l \geqslant 0}$ further satisfies the following relations:

$$
\begin{aligned}
& \sum_{p+r=n} \sum_{\sigma \in \mathfrak{S}_{n}} \frac{(-1)^{\epsilon(\sigma)}}{p ! r !} f_{1+r, m}\left(\left(l_{p} \otimes \mathbf{1}_{c}^{\otimes r} \otimes \mathbf{1}_{o}^{\otimes m}\right)\left(c_{\sigma(1)}, \ldots, c_{\sigma(n)} ; o_{1}, \ldots, o_{m}\right)\right) \\
& \quad+\sum_{p+r=n} \sum_{i+s+j=m} \sum_{\sigma \in \mathfrak{S}_{n}} \frac{(-1)^{\epsilon(\sigma)}}{p ! r !} f_{p, i+1+j}\left(\left(\mathbf{1}_{c}^{\otimes p} \otimes \mathbf{1}_{o}^{\otimes i} \otimes n_{r, s} \otimes \mathbf{1}_{o}^{\otimes j}\right)\left(c_{\sigma(1)}, \ldots, c_{\sigma(n)} ; o_{1}, \ldots, o_{m}\right)\right) \\
& =\sum_{\substack{\left(r_{1}+\cdots+r_{i}\right)+\left(p_{1}+\cdots+p_{j}\right)=n \\
\left(q_{1}+\cdots+q_{j}\right)=m}} \sum_{\sigma \in \mathfrak{S}_{n}} \frac{(-1)^{\epsilon(\sigma)}}{i !\left(r_{1} ! \cdots r_{i} !\right)\left(p_{1} ! \cdots p_{j} !\right)} n_{i, j}^{\prime}\left(\left(f_{r_{1}} \otimes \cdots \otimes f_{r_{i}} \otimes f_{p_{1}, q_{1}}\right.\right. \\
& \left.\left.\quad \otimes \cdots \otimes f_{\left.p_{j}, q_{j}\right)}\right)\left(c_{\sigma(1)}, \ldots, c_{\sigma(n)} ; o_{1}, \ldots, o_{m}\right)\right) .
\end{aligned}
$$

The right-hand side is written explicitly as

$$
\begin{aligned}
n_{i, j}^{\prime}( & \left.\left(f_{r_{1}} \otimes \cdots \otimes f_{r_{i}} \otimes f_{p_{1}, q_{1}} \otimes \cdots \otimes f_{p_{j}, q_{j}}\right)\left(c_{\sigma(1)}, \ldots, c_{\sigma(n)} ; o_{1}, \ldots, o_{m}\right)\right) \\
= & (-1)^{\tau_{\vec{p}, \vec{q}}(\sigma)} n_{i, j}^{\prime}\left(f_{r_{1}}\left(c_{\sigma(1)}, \ldots, c_{\sigma\left(r_{1}\right)}\right), \ldots, f_{r_{i}}\left(c_{\sigma\left(\bar{r}_{i-1}+1\right)}, \ldots, c_{\sigma\left(\bar{r}_{i}\right)}\right) ;\right. \\
& \left.\times f_{p_{1}, q_{1}}\left(c_{\sigma\left(\bar{r}_{i}+1\right)}, \ldots, c_{\sigma\left(\bar{p}_{1}\right)} ; o_{1}, \ldots, o_{q_{1}}\right), \ldots, f_{p_{j}, q_{j}}\left(c_{\sigma\left(\bar{p}_{j-1}+1\right)}, \ldots, c_{\sigma\left(\bar{p}_{j}\right)} ; o_{\bar{q}_{j-1}+1}, \ldots, o_{\bar{q}_{j}}\right)\right),
\end{aligned}
$$

where $\bar{r}_{k}:=r_{1}+\cdots+r_{k}, \bar{p}_{k}:=\bar{r}_{i}+p_{1}+\cdots+p_{k}, \bar{q}_{k}:=q_{1}+\cdots+q_{k}$ and $\tau_{\vec{p}, \vec{q}}(\sigma)$ is given by

$$
\tau_{\vec{p}, \vec{q}}(\sigma)=\sum_{k=1}^{j-1}\left(c_{\sigma\left(\bar{p}_{k}+1\right)}+\cdots+c_{\sigma\left(\bar{p}_{k+1}\right.}\right)\left(o_{1}+\cdots+o_{\bar{q}_{k}}\right) .
$$


In particular, if $(\mathcal{H}, \mathfrak{l}, \mathfrak{n})$ and $\left(\mathcal{H}^{\prime}, \mathfrak{l}^{\prime}, \mathfrak{n}^{\prime}\right)$ are OCHAs and if $f_{0}=f_{0,0}=0$, we call it an OCHA morphism.

Definition 2.11 (quasi-isomorphism): Suppose that two OCHAs $(\mathcal{H}, \mathfrak{l}, \mathfrak{n}),\left(\mathcal{H}^{\prime}, \mathfrak{l}^{\prime}, \mathfrak{n}^{\prime}\right)$ and an OCHA morphism $\mathfrak{f}:(\mathcal{H}, \mathfrak{l}, \mathfrak{n}) \rightarrow\left(\mathcal{H}^{\prime}, \mathfrak{l}^{\prime}, \mathfrak{n}^{\prime}\right)$ are given. $\mathfrak{f}$ is called an open-closed homotopy algebra quasi-isomorphism if $f_{1}: \mathcal{H}_{c} \rightarrow \mathcal{H}_{c}^{\prime}$ induces an isomorphism between the cohomology spaces of the complexes $\left(\mathcal{H}_{c}, l_{1}\right)$ and $\left(\mathcal{H}_{c}^{\prime}, l_{1}^{\prime}\right)$, and further $f_{0,1}: \mathcal{H}_{o} \rightarrow \mathcal{H}_{o}^{\prime}$ induces an isomorphism between the cohomology spaces of the complexes $\left(\mathcal{H}_{o}, n_{0,1}\right)$ and $\left(\mathcal{H}_{o}^{\prime}, n_{0,1}^{\prime}\right)$. In particular, if $f_{1}$ and $f_{0,1}$ are isomorphisms, we call $\mathfrak{f}$ an open-closed homotopy algebra isomorphism.

\section{Cyclic structures in OCHAs}

Now we consider an additional structure, cyclicity, (cf. Ref. 16) on open-closed homotopy algebras. It is defined in terms of constant symplectic inner products. The string theory motivation for this additional structure is that punctures on the boundary of the disk inherit a cyclic order from the orientation of the disk and the operations are to respect this cyclic structure, just as the $L_{\infty}$ structure reflects the symmetry of the punctures in the interior of the disk or on the sphere. Alternatively, a typical Lagrangian of a (quantum) field theory originally has such structure and in particular in the Batalin-Vilkovisky (BV) formalism, ${ }^{3,4}$ such structure is defined in terms of an odd (degree minus one) symplectic structure on the corresponding supermanifold. ${ }^{3,62,1,33}$ Both pictures are then combined with each other in string field theory as discussed in Sec. IV.

From such background, in Ref. 33 a "cyclicity" is defined for $A_{\infty}$ algebras in terms of a degree minus one constant symplectic inner product, and it is shown that homotopy invariant properties of $A_{\infty}$ algebras hold true also in the category of cyclic $A_{\infty}$ algebras. However, in string theory or in particular topological string theory, there often exist cyclic structures defined by inner products having some different degree. For the arguments on homotopy invariant properties in Ref. 33, the degree of the inner product is not essential. Thus, we define cyclic structures with constant symplectic inner products of arbitrary fixed integer degrees.

Definition 2.12 (constant symplectic structure): Bilinear maps, $\omega_{c}: \mathcal{H}_{c} \otimes \mathcal{H}_{c} \rightarrow \mathrm{C}$ and $\omega_{o}: \mathcal{H}_{o}$ $\otimes \mathcal{H}_{o} \rightarrow \mathrm{C}$, are called constant symplectic structures when they have fixed integer degrees $\left|\omega_{c}\right|,\left|\omega_{o}\right| \in \mathbb{Z}$ and are nondegenerate and skew-symmetric. Here skew-symmetric indicates that

$$
\omega_{c}\left(c_{2}, c_{1}\right)=-(-1)^{c_{1} c_{2}} \omega_{c}\left(c_{1}, c_{2}\right), \quad \omega_{o}\left(o_{2}, o_{1}\right)=-(-1)^{o_{1} o_{2}} \omega_{o}\left(o_{1}, o_{2}\right)
$$

for any $c_{1}, c_{2} \in \mathcal{H}_{c}, o_{1}, o_{2} \in \mathcal{H}_{o}$, and the degree of $\omega_{c}, \omega_{o}$ implies that $\omega_{c}\left(c_{1}, c_{2}\right)=0$ except for $\operatorname{deg}\left(c_{1}\right)+\operatorname{deg}\left(c_{2}\right)+\left|\omega_{c}\right|=0$ and $\omega_{o}\left(o_{1}, o_{2}\right)=0$ except for $\operatorname{deg}\left(o_{1}\right)+\operatorname{deg}\left(o_{2}\right)+\left|\omega_{o}\right|=0$. We further denote the constant symplectic structure on $\mathcal{H}=\mathcal{H}_{c} \oplus \mathcal{H}_{o}$ by $\omega:=\omega_{c} \oplus \omega_{o}$.

Suppose that an open-closed homotopy algebra $(\mathcal{H}, \mathfrak{l}, \mathfrak{n})$ is equipped with constant symplectic structures $\omega_{c}: \mathcal{H}_{c} \otimes \mathcal{H}_{c} \rightarrow \mathrm{C}$ and $\omega_{o}: \mathcal{H}_{o} \otimes \mathcal{H}_{o} \rightarrow \mathrm{C}$ as in Definition 2.12.

For $\left\{l_{k}\right\}_{k \geqslant 1}$ and $\left\{n_{p, q}\right\}_{p+q}$, let us define two kinds of multilinear maps by

$$
\mathcal{V}_{k+1}=\omega_{c}\left(l_{k} \otimes \mathbf{1}_{c}\right):\left(\mathcal{H}_{c}\right)^{\otimes(k+1)} \rightarrow \mathrm{C}, \quad \mathcal{V}_{p, q+1}=\omega_{o}\left(n_{p, q} \otimes \mathbf{1}_{o}\right):\left(\mathcal{H}_{c}\right)^{\otimes p} \otimes\left(\mathcal{H}_{o}\right)^{\otimes(q+1)} \rightarrow \mathrm{C}
$$

or more explicitly

$$
\mathcal{V}_{k+1}\left(c_{1}, \ldots, c_{k+1}\right)=\omega_{c}\left(l_{k}\left(c_{1}, \ldots, c_{k}\right), c_{k+1}\right)
$$

and

$$
\mathcal{V}_{p, q+1}\left(c_{1}, \ldots, c_{p} ; o_{1}, \ldots, o_{q+1}\right)=\omega_{o}\left(n_{p, q}\left(c_{1}, \ldots, c_{p} ; o_{1}, \ldots, o_{q}\right), o_{q+1}\right) .
$$

The degree of $\mathcal{V}_{k+1}$ and $\mathcal{V}_{p, q+1}$ are $\left|\omega_{c}\right|+1$ and $\left|\omega_{o}\right|+1$. Note that the degrees of $\mathcal{V}_{k+1}$ and $\mathcal{V}_{p, q+1}$ are zero when they come from odd constant symplectic structures $\left|\omega_{c}\right|=\left|\omega_{o}\right|=-1$.

Definition 2.13 (cyclic open-closed homotopy algebra): An open-closed homotopy algebra $(\mathcal{H}, \omega, \mathfrak{l}, \mathfrak{n})$ is called a cyclic open-closed homotopy algebra (COCHA) when $\mathcal{V}_{k+1}$ is graded symmetric with respect to any permutation of $\left(\mathcal{H}_{c}\right)^{\otimes(k+1)}$ and $\mathcal{V}_{p, q+1}$ has cyclic symmetry with respect to cyclic permutations of $\left(\mathcal{H}_{o}\right)^{\otimes(q+1)}$, that is, if 


$$
\mathcal{V}_{k+1}\left(c_{1}, \ldots, c_{k+1}\right)=(-1)^{\epsilon(\sigma)} \mathcal{V}_{k+1}\left(c_{\sigma(1)}, \ldots, c_{\sigma(k+1)}\right), \quad \sigma \in \mathfrak{S}_{k+1}
$$

and

$$
\mathcal{V}_{p, q+1}\left(c_{1}, \ldots, c_{p} ; o_{1}, \ldots, o_{q+1}\right)=(-1)^{o_{1}\left(o_{2}+\cdots+o_{q+1}\right)} \mathcal{V}_{k+1, l}\left(c_{1}, \ldots, c_{p} ; o_{2}, \ldots, o_{q+1}, o_{1}\right)
$$

The graded commutativity of $\mathcal{V}_{p, q+1}$ with respect to permutations of $\left(\mathcal{H}_{c}\right)^{\otimes p}$, that is,

$$
\mathcal{V}_{p, q+1}\left(c_{1}, \ldots, c_{p} ; o_{1}, \ldots, o_{q+1}\right)=(-1)^{\epsilon(\sigma)} \mathcal{V}_{p, q+1}\left(c_{\sigma(1)}, \ldots, c_{\sigma(p)} ; o_{1}, \ldots, o_{q+1}\right), \quad \sigma \in \mathfrak{S}_{p}
$$

automatically holds by the definition of $\mathfrak{n}$.

Note also that there are many situations where the inner products exist only for open strings. This is the case for the topological string situation in the B-model we will discuss later in Sec. VI C. For instance, on the topological open string side, there often exists a natural inner product coming essentially from an integral (trace) of products of two differential forms. The inner products of this kind in fact turn out to be skew-symmetric (symplectic) in our suspended notation (see Ref. 34). See also Ref. 57 for more general cyclic structures including nonskew inner products.

On the other hand, if we have $\omega_{c}$ and $\omega_{o}$, nondegenerate inner products in both open and closed string sides, we can identify $\mathcal{H}$ with its linear dual, then reverse the process and define further maps

$$
r_{p-1, q+1}:\left(\mathcal{H}_{c}\right)^{\otimes(p-1)} \otimes\left(\mathcal{H}_{o}\right)^{\otimes(q+1)} \rightarrow \mathcal{H}_{c}
$$

with relations amongst themselves and with the operations already defined, which can easily be deduced from their definition. In particular, for $n_{1,0}: \mathcal{H}_{c} \rightarrow \mathcal{H}_{o}$ we have $r_{0,1}: \mathcal{H}_{o} \rightarrow \mathcal{H}_{c}$. Namely, for the cyclic case the fundamental object is the multilinear map $\mathcal{V}_{p, q+1}$ where $n_{p, q}$ and $r_{p-1, q+1}$ are equivalent under the relation above.

Physically, the multilinear map $\mathcal{V}_{p, q+1}$ is related to the (scattering) amplitudes of a disk with $p$ closed strings and $(q+1)$ open strings insertions. Choosing an open string state as a root edge instead of a closed string state, that is, taking $n_{p, q}$ instead of $r_{p-1, q+1}$, for defining an OCHA is related to a standard compactification of the moduli spaces of the corresponding Riemann surface [a disk with $p$ points interior and $(q+1)$ points on the boundary]. Also, in the next section we shall see that, due to this choice of the root edge, the OCHA structure $(\mathfrak{l}, \mathfrak{n})$ can be singled out to be an odd vector field on the appropriate supermanifold.

Remark 2.14(Category version): As an $A_{\infty}$ category is defined as a straightforward extension of an $A_{\infty}$ algebra, ${ }^{9}$ one can extend our open-closed homotopy algebra to its category version by replacing $\mathcal{H}_{o}$ by the space of morphisms of a category. This category extension corresponds to considering many D-branes on which open strings end. This is important for applying OCHAs to topological string theory, see Sec. VI C.

\section{THE DUAL SUPERMANIFOLD DESCRIPTION}

\section{A. OCHAs and odd formal vector fields}

For a graded vector space $\mathcal{H}=\mathcal{H}_{c} \oplus \mathcal{H}_{o}$, denote by $\left\{\mathbf{e}_{c, i}\right\}$ a basis of $\mathcal{H}_{c}$ and by $\left\{\mathbf{e}_{o, i}\right\}$ a basis of $\mathcal{H}_{o}$. For each $\mathbf{e}_{c, i} \in \mathcal{H}_{c}$ represent the dual base as $\psi^{i}$ and similarly the dual base of $\mathbf{e}_{o, i} \in \mathcal{H}_{o}$ as $\phi^{i}$. We set the degree of the dual basis by $\operatorname{deg}\left(\psi^{i}\right)=-\operatorname{deg}\left(\mathbf{e}_{c, i}\right)$ and $\operatorname{deg}\left(\phi^{i}\right)=-\operatorname{deg}\left(\mathbf{e}_{o, i}\right)$. We consider the formal power series ring in the variables $\left\{\phi^{i}\right\},\left\{\psi^{i}\right\}$, and $\left\{\phi^{i}\right\} \amalg\left\{\psi^{i}\right\}$, and denote them by $C(\phi)$, $C(\psi)$, and $C(\psi, \phi)$, respectively. We define $\left\{\psi^{i}\right\}$ to be associative and graded commutative and $\left\{\phi^{i}\right\}$ to be associative but noncommutative. More precisely, in the space of the formal power series of associative fields $\left\{\phi^{i}\right\} \amalg\left\{\psi^{i}\right\}$, an element in $\left\{\psi^{i}\right\}$ is graded commutative with respect to all elements. Therefore, any element in $C(\psi, \phi)$ is represented as

$$
a(\psi, \phi)=\sum_{k, l} a_{i_{1} \cdots i_{k} ; j_{1} \cdots j_{l}}\left(\phi^{j_{l}} \cdots \phi^{j_{1}}\right)\left(\psi^{i_{k}} \cdots \psi^{i_{1}}\right),
$$

where the coefficient $a_{i_{1} \cdots i_{k} ; j_{1} \cdots j_{l}} \in \mathrm{C}$ is graded symmetric with respect to $i_{1} \cdots i_{k}$. We can call 
$(\mathcal{H}, C(\phi, \psi))$ the formal supermanifold ${ }^{1,40}$ corresponding to an OCHA $(\mathcal{H}, \mathfrak{l}, \mathfrak{n})$. Though usually the term super indicates $\mathbb{Z}_{2}$ graded, we use it for $\mathbb{Z}$ graded objects. On the other hand, an $A_{\infty}$ algebra is described on a formal noncommutative supermanifold $\left(\mathcal{H}_{o}, C(\phi)\right),{ }^{31,33}$ and an $L_{\infty}$ algebra is described on a formal graded commutative supermanifold $\left(\mathcal{H}_{c}, C(\psi)\right)$.

For a weak OCHA $(\mathcal{H}, \mathfrak{l}, \mathfrak{n})$, express the collection of multilinear maps

$$
l_{k}:\left(\mathcal{H}_{c}\right)^{\otimes k} \rightarrow \mathcal{H}_{c}, \quad n_{k, l}:\left(\mathcal{H}_{c}\right)^{\otimes k} \otimes\left(\mathcal{H}_{o}\right)^{\otimes l} \rightarrow \mathcal{H}_{o},
$$

in terms of the bases,

$$
\begin{gathered}
l_{k}\left(\mathbf{e}_{c, i_{1}}, \ldots, \mathbf{e}_{c, i_{k}}\right)=\mathbf{e}_{c, j} c_{i_{1} \cdots i_{k}}^{j}, \quad c_{i_{1} \cdots i_{k}}^{j} \in \mathbb{C}, \\
n_{k, l}\left(\mathbf{e}_{c, i_{1}}, \ldots, \mathbf{e}_{c, i_{k}} ; \mathbf{e}_{o, j_{1}}, \ldots, \mathbf{e}_{o, j_{l}}\right)=\mathbf{e}_{o, j} c_{i_{1} \cdots i_{k} ; j_{1} \cdots j_{l}}^{j}, \quad c_{i_{1} \cdots i_{k} ; j_{1} \cdots j_{l}}^{j} \in \mathrm{C} .
\end{gathered}
$$

Correspondingly, let us define an odd formal vector field $\delta:=\delta_{S}+\delta_{D}: C(\phi, \psi) \rightarrow C(\phi, \psi)$, where

$$
\begin{gathered}
\delta_{S}=\frac{\overleftarrow{\partial}}{\partial \psi^{j}} c^{j}(\psi)=\sum_{k \geqslant 0} \frac{1}{k !} \frac{\overleftarrow{\partial}}{\partial \psi^{j}} c_{i_{1} \cdots i_{k}}^{j} \psi^{i_{k} \cdots} \psi^{i_{1}}, \quad c^{j}(\psi) \in C(\psi), \\
\delta_{D}=\frac{\overleftarrow{\partial}}{\partial \phi^{j}} c^{j}(\phi, \psi)=\sum_{k+l \geqslant 0} \frac{1}{k !} \frac{\overleftarrow{\partial}}{\partial \phi^{j}} c_{i_{1} \cdots i_{k}, j_{1} \cdots j_{l}}^{j}\left(\phi^{j_{l} \cdots} \phi^{j_{1}}\right)\left(\psi^{j_{k} \cdots} \psi^{i_{1}}\right), \quad c^{j}(\phi, \psi) \in C(\phi, \psi) .
\end{gathered}
$$

We use right derivatives just for the sign problem; it is easy to relate this dual supermanifold description to the convention in the preceding section. Since $\mathfrak{l}$ and $\mathfrak{n}$ have degree one, $\delta$ also has degree one.

It acts on $C(\phi, \psi)$ as follows:

$$
\begin{aligned}
\delta(a(\phi, \psi))= & \sum_{k, l} a_{i_{1} \cdots i_{k} ; j_{1} \cdots j_{l}} \sum_{s=1}^{k}(-1)^{\beta_{S}(s-1)}\left(\phi^{j_{l}} \cdots \phi^{j_{1}}\right)\left(\psi^{j_{k}} \cdots \delta_{S}\left(\psi^{i_{s}}\right) \cdots \psi^{i_{1}}\right) \\
& +\sum_{k, l} a_{i_{1} \cdots i_{k} ; j_{1} \cdots j_{l}} \sum_{t=1}^{l}(-1)^{\beta_{D}(t-1)}\left(\phi^{j_{l}} \cdots \delta_{D}\left(\phi^{j_{t}}\right) \cdots \phi^{j_{1}}\right)\left(\psi^{j_{k}} \cdots \psi^{i_{1}}\right),
\end{aligned}
$$

where $\delta_{D}\left(\phi^{j_{t}}\right)=c^{j_{t}}(\phi, \psi)$ and $\delta_{S}\left(\psi^{i_{s}}\right)=c^{i_{s}}(\psi)$. Then, $\beta_{S}(s-1)$ (respectively, $\left.\beta_{D}(t-1)\right)$ is the sign arising when $\delta_{S}$ (respectively, $\delta_{D}$ ) acts from the right and passes the corresponding superfields and is given explicitly by

$$
\beta_{S}(s-1)=\mathbf{e}_{c, i_{1}}+\cdots+\mathbf{e}_{c, i_{s-1}}, \quad \beta_{D}(t-1)=\left(\mathbf{e}_{c, i_{1}}+\cdots+\mathbf{e}_{c, i_{k}}\right)+\mathbf{e}_{o, j_{1}}+\cdots+\mathbf{e}_{o, j_{t-1}} .
$$

The above $\delta(a(\phi, \psi))$ is further rewritten; in the first line $\psi$ 's in $\delta_{D}\left(\phi^{j_{t}}\right)$ are brought to the right of $\phi$ 's, and $\psi$ 's on each line of Eq. (3.3) are treated as graded symmetric. The $\delta(a(\phi, \psi))$ is expressed in the form in Eq. (3.1) again but with a different coefficient. In the supermanifold language, $\delta$ is called an (odd) formal vector field on the formal supermanifold. A formal manifold with such a $\delta$ is called a $Q$-manifold in Ref. 1 if $Q=\delta$ with $Q^{2}=0$.

Lemma 3.1: The condition that $(\mathcal{H}, \mathfrak{l}, \mathfrak{n})$ is a weak OCHA is equivalent to

$$
(\delta)^{2}=0 .
$$

In particular, $\delta$ defines an OCHA if the $k=0$ part of $\delta_{S}$ and $k=l=0$ part of $\delta_{D}$ in Eq. (3.2) are absent or zero. 
The equation above can be expanded as $\left(\delta_{S}\right)^{2}+\left[\delta_{S}, \delta_{D}\right]+\left(\delta_{D}\right)^{2}=0$, where $\left[\delta_{S}, \delta_{D}\right]=\delta_{S}\left(\delta_{D}\right)$ $+\delta_{D}\left(\delta_{S}\right), \delta_{S}\left(\delta_{D}\right)=\left(\grave{\partial} / \partial \phi^{j}\right)\left(\delta_{S}\left(c^{j}(\phi, \psi)\right)\right)$. Note that $\delta_{D}\left(\delta_{S}\right)$ vanishes since $\delta_{S}$ does not include $\phi$. Furthermore, one can see that

$$
\left(\delta_{S}\right)^{2}=0, \quad \delta_{S}\left(\delta_{D}\right)+\left(\delta_{D}\right)^{2}=0
$$

hold independently. The first one is just the dual of the $L_{\infty}$ condition (2.4), and the second one is the dual description of the OCHA condition (2.6). The pair of the equations above can also be thought of as a deformation of $\delta_{S}$ by $\delta_{S}+\delta_{D}$, though we do not discuss this type of deformation in this paper.

A (weak) OCHA morphism in Definition 2.10 can also be rewritten in the same way. For the collection $\mathfrak{f}$ of degree zero multilinear maps,

$$
f_{l}:\left(\mathcal{H}_{c}\right)^{\otimes l} \rightarrow \mathcal{H}_{c}^{\prime}, \quad f_{k, l}:\left(\mathcal{H}_{c}\right)^{\otimes k} \otimes\left(\mathcal{H}_{o}\right)^{\otimes l} \rightarrow \mathcal{H}_{o}^{\prime},
$$

let us now express $f_{k}$ and $f_{k, l}$ as

$$
\begin{gathered}
f_{k}\left(\mathbf{e}_{c, i_{1}}, \ldots, \mathbf{e}_{c, i_{k}}\right)=\mathbf{e}_{c, j^{\prime}} f_{i_{1} \cdots i_{k}}^{j^{\prime}}, \quad f_{i_{1} \cdots i_{k}}^{j^{\prime}} \in \mathrm{C}, \\
f_{k, l}\left(\mathbf{e}_{c, i_{1}}, \ldots, \mathbf{e}_{c, i_{k}} ; \mathbf{e}_{o, j_{1}}, \ldots, \mathbf{e}_{o, j_{l}}\right)=\mathbf{e}_{o, j^{\prime}} j_{i_{1} \cdots i_{k} ; j_{1} \cdots j_{l}}^{j^{\prime}}, \quad f_{i_{1} \cdots i_{k} ; j_{1} \cdots j_{l}}^{j^{\prime}} \in \mathrm{C} .
\end{gathered}
$$

They define the following coordinate transformation between the two supermanifolds $(\mathcal{H}, \mathfrak{l}, \mathfrak{n})$ and $\left(\mathcal{H}^{\prime}, \mathfrak{l}^{\prime}, \mathfrak{n}^{\prime}\right)$ :

$$
\begin{aligned}
& \psi^{j^{\prime}}=\mathfrak{f}_{*}^{j^{\prime}}(\psi)=f^{j^{\prime}}+f_{i}^{j^{\prime}} \psi^{i}+f_{i_{1} i_{2}}^{j^{\prime}} \psi^{j_{2}} \psi^{\dot{j}_{1}}+\cdots+f_{i_{1} \cdots i_{n}}^{j^{\prime}} \psi^{j_{n}} \cdots \psi^{i_{1}}+\cdots, \\
& \phi^{j^{\prime}}=\mathfrak{f}_{*}^{j^{\prime}}(\phi, \psi)=\sum_{k, l \geqslant 0} f_{i_{1} \cdots i_{k} ; j_{1} \cdots j_{l}}^{j^{\prime}}\left(\phi^{j_{l}} \cdots \phi^{j_{1}}\right)\left(\psi^{i_{k}} \cdots \psi^{i_{1}}\right) .
\end{aligned}
$$

This induces a pullback from $C\left(\phi^{\prime}, \psi^{\prime}\right)$ to $C(\phi, \psi)$,

$$
\mathfrak{f}^{*}\left(a\left(\phi^{\prime}, \psi^{\prime}\right)\right)=a\left(\mathfrak{f}_{*}(\phi, \psi), \mathfrak{f}_{*}(\psi)\right),
$$

where $\left\{\phi^{i}\right\}$ and $\left\{\phi^{i^{\prime}}\right\}$ are the coordinates on $\mathcal{H}$ and $\mathcal{H}^{\prime}$, respectively.

Lemma 3.2: The condition that this $f$ is a weak OCHA morphism is then that the map between two formal supermanifolds $\mathfrak{f}_{*}$ is compatible with the actions of $\delta$ and $\delta^{\prime}$ on both sides, that is,

$$
f^{*} \delta^{\prime}\left(a\left(\phi^{\prime}, \psi^{\prime}\right)\right)=\delta f^{*} a\left(\phi^{\prime}, \psi^{\prime}\right)
$$

holds for any $a\left(\phi^{\prime}, \psi^{\prime}\right) \in C\left(\phi^{\prime}, \psi^{\prime}\right)$. In other words, $\mathfrak{f}_{*}$ is a morphism between $Q$ manifolds. If in addition $f^{j^{\prime}}=f_{\varnothing ; \varnothing}^{j^{\prime}}=0, \mathfrak{f}_{*}$ preserves the origin of the formal supermanifolds. $\mathfrak{f}_{*}$ is then an OCHA morphism in the situation that both $\delta$ and $\delta^{\prime}$ define OCHAs.

All these structures in the supermanifold description are dual to the coalgebra description explained in Ref. 35 in the following sense (see Ref. 33 for the $A_{\infty}$ case). Let us introduce natural pairings

$$
\left\langle\psi^{i} \mid \mathbf{e}_{c, j}\right\rangle=\delta_{j}^{i}, \quad\left\langle\phi^{i} \mid \mathbf{e}_{o, j}\right\rangle=\delta_{j}^{i}
$$

and also the extended pairings

$$
\left\langle\left(\phi^{j_{l}} \cdots \phi^{j_{1}}\right)\left(\psi^{i_{k}} \cdots \psi^{i_{1}}\right) \mid\left(\mathbf{e}_{c, i_{1}^{\prime}} \cdots \mathbf{e}_{c, i_{k}^{\prime}}\right) \otimes\left(\mathbf{e}_{o, j_{1}^{\prime}} \cdots \mathbf{e}_{o, j_{l}^{\prime}}\right)\right\rangle=\epsilon_{i_{1}^{\prime} \cdots i_{k}^{\prime}}^{i_{1} \cdots i_{k}} \delta_{j_{1}^{\prime}}^{j_{1}} \cdots \delta_{j_{k}^{\prime}}^{j_{k}}
$$

for $k+l>1$, where $\epsilon_{i_{1}^{\prime} \cdots i_{k}^{\prime}}^{i_{1} \cdots i_{k}}:=\Sigma_{\sigma \in \mathfrak{S}_{k}} \epsilon(\sigma) \delta_{i_{1}^{\prime}}^{i_{\sigma(1)}} \cdots \delta_{i_{k}^{\prime}}^{i_{\sigma(k)}}$. We set the pairing to be zero if the number of elements $\psi / \phi$ and $\mathbf{e}_{c} / \mathbf{e}_{o}$ does not coincide. The space spanned by $\left(\mathbf{e}_{c, i_{1}} \cdots \mathbf{e}_{c, i_{k}}\right) \otimes\left(\mathbf{e}_{o, j_{1}} \cdots \mathbf{e}_{o, j_{l}}\right), k$ $+l \geqslant 1$, is what is denoted $C\left(\mathcal{H}_{c}\right) \otimes T^{c}\left(\mathcal{H}_{o}\right)$ in Ref. 35. As the adjoint of the product on $C(\psi, \phi)$, a 
coproduct $\quad \Delta: C\left(\mathcal{H}_{c}\right) \otimes T^{c}\left(\mathcal{H}_{o}\right) \rightarrow\left(C\left(\mathcal{H}_{c}\right) \otimes T^{c}\left(\mathcal{H}_{o}\right)\right) \otimes\left(C\left(\mathcal{H}_{c}\right) \otimes T^{c}\left(\mathcal{H}_{o}\right)\right) \quad$ is $\quad$ defined; for $\quad a, b$ $\in C(\psi, \phi)$ and $x \in C\left(\mathcal{H}_{c}\right) \otimes T^{c}\left(\mathcal{H}_{o}\right)$,

$$
\langle b \cdot a \mid x\rangle=\sum_{i}\left\langle a \mid x_{i}^{+}\right\rangle \cdot\left\langle b \mid x_{i}^{-}\right\rangle, \quad \Delta:=\sum_{i}\left(x_{i}^{+} \otimes x_{i}^{-}\right) .
$$

Thus, $C\left(\mathcal{H}_{c}\right) \otimes T^{c}\left(\mathcal{H}_{o}\right)$ forms a coalgebra. Then, a codifferential $\mathfrak{l}+\mathfrak{n}: C\left(\mathcal{H}_{c}\right) \otimes T^{c}\left(\mathcal{H}_{o}\right) \rightarrow C\left(\mathcal{H}_{c}\right)$ $\otimes T^{c}\left(\mathcal{H}_{o}\right)$ is given as the adjoint of $\delta=\delta_{S}+\delta_{D}$,

$$
\langle a \mid(\mathfrak{l}+\mathfrak{n})(x)\rangle:=\langle\delta(a) \mid x\rangle .
$$

In a similar way, a coalgebra homomorphism $\mathfrak{f}: C\left(\mathcal{H}_{c}\right) \otimes T^{c}\left(\mathcal{H}_{o}\right) \rightarrow C\left(\mathcal{H}_{c}^{\prime}\right) \otimes T^{c}\left(\mathcal{H}_{o}^{\prime}\right)$ is obtained as the adjoint of the pullback $\mathfrak{f}^{*}: C\left(\phi^{\prime}, \psi^{\prime}\right) \rightarrow C(\phi, \psi)$. Thus, the coalgebra description given in Ref. 35 is obtained as the dual of the algebra description in terms of formal power series on supermanifolds.

\section{B. Cyclicity and constant symplectic and/or Poisson structures}

Next, we discuss the cyclicity (Definition 2.13). If cyclicity is imposed on the $\phi^{i}$, s, we indicate that by $C(\phi)_{\text {cyc }}$ or $C(\phi, \psi)_{\text {cyc }}$. Any element of $C(\phi, \psi)_{\text {cyc }} \subset C(\phi, \psi)$ is represented in the form in Eq. (3.1) but the coefficient $a_{i_{1} \cdots i_{k} ; j_{1} \cdots j_{l}}$ is in addition graded cyclic symmetric with respect to the indices $j_{1} \cdots j_{l}$. On this algebra, a constant Poisson structure is introduced naturally by dualizing the constant symplectic structures in Definition 2.12.

Definition 3.3 (constant Poisson structure): Suppose $\mathcal{H}_{c}$ and $\mathcal{H}_{o}$ have constant symplectic structures $\omega_{c}: \mathcal{H}_{c} \otimes \mathcal{H}_{c} \rightarrow \mathrm{C}$ and $\omega_{o}: \mathcal{H}_{o} \otimes \mathcal{H}_{o} \rightarrow \mathrm{C}$ (Definition 2.12). The corresponding Poisson brackets are denoted by

$$
(,)_{c}=\frac{\overleftarrow{\partial}}{\partial \phi^{i}} \omega_{c}^{i j} \frac{\vec{\partial}}{\partial \phi^{j}}, \quad(,)_{o}=\frac{\overleftarrow{\partial}}{\partial \phi^{i}} \omega_{o}^{i j} \frac{\vec{\partial}}{\partial \phi^{j}}
$$

Here $\omega_{c}^{i j} \in \mathbb{C}$ and $\omega_{o}^{i j} \in \mathbb{C}$ are the inverse matrices of $\omega_{c, i j}:=\omega_{c}\left(\mathbf{e}_{c, i}, \mathbf{e}_{c, j}\right)$ and $\omega_{o, i j}:=\omega_{o}\left(\mathbf{e}_{o, i}, \mathbf{e}_{o, j}\right)$. That is, $\omega_{c, i j} \omega_{c}^{j k}=\omega_{c}^{k j} \omega_{c, j i}=\delta_{i}^{k}$ and $\omega_{o, i j} \omega_{o}^{j k}=\omega_{o}^{k j} \omega_{o, j i}=\delta_{i}^{k}$ hold. Thus (, $)_{c}$ is a graded Poisson bracket for a graded commutative algebra and $(,)_{o}$ is a Poisson bracket for the cyclic algebra as in Ref. 31. $C(\psi)_{\text {cyc }}$ and $C(\phi)_{\text {cyc }}$ form graded Poisson algebras with Poisson brackets $(,)_{c}$ and $(,)_{o}$, respectively. Furthermore, these two Poisson brackets can be combined naturally and extended to one on $C(\phi, \psi)_{\mathrm{cyc}}$.

A COCHA (Definition 2.13) is dualized as follows. For the collection of multilinear maps $\mathcal{V}_{k}$ and $\mathcal{V}_{k, l}$, let us define their coefficients by

$$
\mathcal{V}_{k}\left(\mathbf{e}_{c, i_{1}}, \ldots, \mathbf{e}_{c, i_{k}}\right):=\mathcal{V}_{i_{1} \cdots i_{k}} \in \mathbb{C}, \quad \mathcal{V}_{k, l}\left(\mathbf{e}_{c, i_{1}}, \ldots, \mathbf{e}_{c, i_{k}} ; \mathbf{e}_{o, j_{1}}, \ldots, \mathbf{e}_{o, j_{l}}\right):=\mathcal{V}_{i_{1} \cdots i_{k} ; j_{1} \cdots j_{l}} \in \mathbb{C} .
$$

Note that they are graded symmetric with respect to the indices $i_{1} \cdots i_{k}$ and cyclic with respect to the indices $j_{1} \cdots j_{l}$. Consider further a formal sum of polynomial functions $S$,

$$
S(\phi, \psi)=S_{S}(\psi)+S_{D}(\phi, \psi), \quad S_{S}(\psi) \in C(\psi), \quad S_{D}(\phi, \psi) \in C(\phi, \psi)_{\mathrm{cyc}},
$$

where $S_{S}$ and $S_{D}$ are defined by

$$
\begin{aligned}
& S_{S}(\psi)=\sum_{l \geqslant 2} \frac{1}{l !} \mathcal{V}_{i_{1} \cdots i_{l}} \psi^{i_{l}} \cdots \psi^{i_{1}}, \quad \mathcal{V}_{i_{1} \cdots i_{l}} \in \mathrm{C}, \\
& S_{D}(\phi, \psi)=\sum_{k \geqslant 0, l \geqslant 1, k+l \geqslant 2} \frac{1}{k ! l} \mathcal{V}_{i_{1} \cdots i_{k} ; j_{1} \cdots j_{l}}\left(\phi^{j_{l}} \cdots \phi^{j_{1}}\right)\left(\psi^{i_{k}} \cdots \psi^{i_{1}}\right), \quad \mathcal{V}_{i_{1} \cdots i_{k} ; j_{1} \cdots j_{l}} \in \mathrm{C} .
\end{aligned}
$$

Then one can define the formal vector field $\delta$ acting on $C(\phi, \psi)_{\text {cyc }}$ as follows: 


$$
\delta:=\delta_{S}+\delta_{D}, \quad \delta_{S}=\left(, S_{S}\right)_{c}, \quad \delta_{D}=\left(, S_{D}\right)_{o} .
$$

The condition $(\delta)^{2}=0$ coincides with the condition that $(\mathcal{H}, \mathfrak{l}, \mathfrak{n})$ is a COCHA.

\section{ZWIEBACH'S OPEN-CLOSED STRING FIELD THEORY}

String field theory is defined on a fixed conformal background of a space-time (target space) $M$ to which world sheets of strings (Riemann surfaces) are mapped, where the conformal background is a background (metric, etc., of $M$ ) in which string world sheet theory has conformal symmetry. There exist several classes of string field theories corresponding to the classes of Riemann surfaces. The most general one is quantum open-closed string field theory, ${ }^{74}$ which is associated to the most general class of Riemann surfaces: Riemann surfaces of arbitrary genus, possibly with boundaries and punctures.

It includes various substring field theories: classical open string field theories-associated to disks (one boundary and zero genus) with punctures only on the boundary, classical closed string field theories-associated to spheres (no boundary and genus zero) with punctures, quantum closed string field theories-associated to Riemann surfaces with punctures (and various genera) and without boundary, and so on. Genera and multiboundaries relate to closed and open string loops (in the sense of Feynman diagrams), respectively. We use the term classical (respectively, quantum) for theory without loops (respectively, with loops). In this section we shall explain that, extracting the tree open-closed part from Zwiebach's quantum open-closed string field theory, ${ }^{74}$ one obtains an OCHA. Namely, an OCHA is a general homotopy algebraic structure for tree open-closed string field theory as $L_{\infty}$ algebras (respectively, $A_{\infty}$ algebras) are for tree closed (respectively, open) string field theories.

The quantum open-closed string field theory discussed by Zwiebach ${ }^{74}$ is defined by all possible open-closed interaction vertices together with closed and open string kinetic terms satisfying the quantum BV master equation. The interaction term is expressed formally in the following form [Eq. (5.7) of Ref. 74]:

$$
f\left(\mathcal{V}_{b, m}^{g, n}\right)=\left[\frac{1}{n !} \frac{1}{b !} \prod_{k=1}^{b} \frac{1}{m_{k}}\right] \int_{\mathcal{V}_{b, n}^{g, n}}\left\langle\Omega|| \frac{|\Psi\rangle \cdot|\Psi\rangle}{n} \prod_{k=1}^{b} \frac{|\Phi\rangle \cdots|\Phi\rangle}{m_{k}} .\right.
$$

Here the kets $|\Psi\rangle \in \mathcal{H}_{c}$ and $|\Phi\rangle \in \mathcal{H}_{o}$ are the closed string fields and the open string fields, respectively. $\mathcal{V}_{b, m}^{g, n} \subset \overline{\mathcal{M}}_{b, m}^{g, n}$ is the appropriate subspace of the compactified moduli space of Riemann surfaces with genus $g, n$-interior punctures and $b$ boundaries $S^{1}$ having $m_{1}, \ldots, m_{b}$ punctures on them. Equivalently, it has $n$-closed string punctures and $m_{i}$-open string insertions on the corresponding boundary $S^{1}$. The bra $\langle\Omega|$ denotes a differential form on $\overline{\mathcal{M}}_{b, m}^{g, n}$ which takes its value in $\left(\mathcal{H}_{c}^{*}\right)^{\otimes n} \otimes\left(\mathcal{H}_{o}^{*}\right)^{\otimes m_{1}} \otimes \cdots \otimes\left(\mathcal{H}_{o}^{*}\right)^{\otimes m_{b}}$. This data is determined by the conformal field theory for a fixed conformal background. Then, the combination $\int_{\mathcal{V}_{b, m}^{g, n}}\langle\Omega|$ defines a map

$$
\int_{\mathcal{V}_{b, m}^{g, n}}\langle\Omega|:\left(\mathcal{H}_{c}\right)^{\otimes n} \otimes\left(\mathcal{H}_{o}\right)^{\otimes m_{1}} \otimes \cdots \otimes\left(\mathcal{H}_{o}\right)^{\otimes m_{b}} \rightarrow \mathrm{C} .
$$

In terms of bases $\mathbf{e}_{c, i}$ and $\mathbf{e}_{o, j}$ of the spaces of states $\mathcal{H}_{c}$ and $\mathcal{H}_{o}$, the kets can be expressed as $|\Psi\rangle:=\sum_{i} \mathbf{e}_{c, i} \psi^{i}$ and $|\Phi\rangle:=\sum_{j} \mathbf{e}_{o, j} \phi^{j}$, and the coordinates $\psi^{i}$ and $\phi^{i}$ play the role of fields. The degree of each basis element $\mathbf{e}_{c, i}$ or $\mathbf{e}_{o, j}$ is determined by the corresponding conformal field theory on the string world sheet and is related to the degree of field $\psi^{i}$ or $\phi^{j}$ through the relations $\operatorname{deg}\left(\psi^{i}\right)$ $=-\operatorname{deg}\left(\mathbf{e}_{c, i}\right)$ and $\operatorname{deg}\left(\phi^{i}\right)=-\operatorname{deg}\left(\mathbf{e}_{o, i}\right)$. The degrees $\operatorname{deg}\left(\psi^{i}\right)$ and $\operatorname{deg}\left(\phi^{i}\right)$ in turn denote the ghost numbers in the sense of the BV-formalism for the target space field theory. The map (4.2) is defined to be of degree zero because of a ghost number preserving condition on the string world sheets, naturally extended to the polynomials of $\psi^{i}$ and $\phi^{j}$. Then $f\left(\mathcal{V}_{b, m}^{g, n}\right)$ in Eq. (4.1), which is the image of $|\Psi\rangle^{\otimes n} \otimes \Pi_{k=1}^{b}|\Phi\rangle^{\otimes m_{k}}$ by the map (4.2), belongs to a subspace of $C(\psi, \phi)$ whose elements are expressed in general in the form 


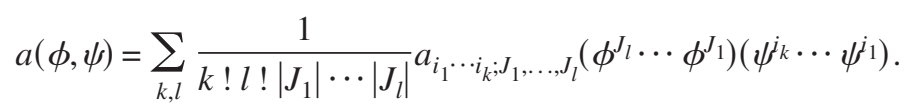

For the interaction terms, $\left|J_{i}\right|=m_{i}$ in the notation in Eq. (4.1). Here $J=\left(j_{1}, \ldots, j_{|J|}\right)$ is the multiindex, $\phi^{J}=\phi^{j|J|} \cdots \phi^{j_{1}}$. The coefficient $a_{i_{1} \cdots i_{k} ; J_{1}, \cdots, J_{l}} \in \mathrm{C}$ is then graded symmetric with respect to the cyclic permutations of each multi-index $J=\left(j_{1}, \ldots, j_{|J|}\right)$, all the permutations of $i_{1} \cdots i_{k}$, and those of $J_{1}, \ldots, J_{l}$. We denote the corresponding subspace by $C(\phi, \psi)_{\mathrm{qoc}}:=C_{\mathrm{sym}}(\psi)$ $\otimes C_{\text {sym }}\left(C(\phi)_{\text {cyc }}\right) \subset C(\phi, \psi)$, where qoc indicates quantum open-closed. Note that, by construction, the degree of $f\left(\mathcal{V}_{b, m}^{g, n}\right)$ is zero. The closed string kinetic term and the open string kinetic term are expressed as follows:

$$
\frac{1}{2}\left\langle\Psi, Q_{c} \Psi\right\rangle_{c}, \quad \frac{1}{2}\left\langle\Phi, Q_{o} \Phi\right\rangle_{o}
$$

which also belong to $C(\phi, \psi)_{\text {qoc }}$ and have degree zero. In our notation, $Q_{c}=l_{1}: \mathcal{H}_{c} \rightarrow \mathcal{H}_{c}$ and $Q_{o}$ $=n_{0,1}: \mathcal{H}_{o} \rightarrow \mathcal{H}_{o}$. Physically, $Q_{c}$ (respectively, $Q_{o}$ ) is called the BRST operator for closed (respectively, open) strings, where BRST is taken in the sense of the conformal field theory on the string world sheet. Their cohomologies then define the physical state spaces of strings. Also, the brackets are just the constant symplectic structures in Definition 2.12,

$$
\langle,\rangle_{c}=\omega_{c}: \mathcal{H}_{c} \otimes \mathcal{H}_{c} \rightarrow \mathrm{C}, \quad\langle,\rangle_{o}=\omega_{o}: \mathcal{H}_{o} \otimes \mathcal{H}_{o} \rightarrow \mathrm{C} .
$$

Since these constant symplectic structures come from the BV formalism ${ }^{3,4}$ in which string field theories are described, the degrees of $\omega_{c}$ and $\omega_{o}$ are set to be minus one. In such a superfield description of the BV formalism, they are called odd symplectic structures ${ }^{62,1}$ since degree minus one implies odd in $\mathbb{Z}_{2}$ grading. The corresponding odd Poisson brackets

$$
(,)=(,)_{c}+(,)_{o}, \quad(,)_{c}=\frac{\overleftarrow{\partial}}{\partial \psi^{j}} \omega_{c}^{i j} \frac{\vec{\partial}}{\partial \psi^{j}}, \quad(,)_{o}=\frac{\overleftarrow{\partial}}{\partial \phi^{i}} \omega_{o}^{i j} \frac{\vec{\partial}}{\partial \phi^{j}}
$$

are what are called the $B V$ brackets. Since they have degree one, $\left(\psi^{i}, \psi^{j}\right)_{c}=\omega_{c}^{i j} \neq 0$ only when the sum of the degree of $\psi^{i}$ and the degree of $\psi^{j}$ is equal to minus one. In the BV formalism, ${ }^{3,4}$ two fields $\psi^{i}$ and $\psi^{j}$ having nonzero $\omega_{c}^{i j}$ make a pair of a field and an antifield. Of course these facts hold true similarly for open string fields (see Refs. 31 and 33). Both Poisson brackets are naturally combined and extended to ( , ) on $C(\phi, \psi)_{\text {qoc }}$. Also, define second order operators as

$$
\Delta=\Delta_{c}+\Delta_{o}, \quad \Delta_{c}=\frac{1}{2}(-1)^{\mathbf{e}_{c, i}} \omega_{c}^{i j} \frac{\vec{\partial}}{\partial \psi^{i}} \frac{\vec{\partial}}{\partial \psi^{j}}, \quad \Delta_{o}=\frac{1}{2}(-1)^{\mathbf{e}_{o, i}} \omega_{o}^{i j} \frac{\vec{\partial}}{\partial \phi^{i}} \frac{\vec{\partial}}{\partial \phi^{j}} .
$$

Since the BV brackets have degree one, we have $\operatorname{deg}(\Delta)=1$, while $\left(C(\psi), \Delta_{c}, \bullet,(,)_{c}\right)$ and $\left(C(\phi)_{\mathrm{cyc}}, \Delta_{o}, \bullet,(,)_{o}\right)$ form BV algebras (see Refs. 3, 4, and 14).

Further $\left(C(\psi, \phi)_{\mathrm{qoc}}, \Delta, \bullet,(),\right)$ forms a BV algebra, where $\bullet: C(\psi, \phi)_{\mathrm{qoc}} \otimes C(\psi, \phi)_{\text {qoc }}$ $\rightarrow C(\psi, \phi)_{\text {qoc }}$ is the associative product, symmetric in the $\psi$ 's. We shall soon reduce them to the tree open-closed structures, so do not stress to explain the detail of the structure on the whole $C(\psi, \phi)_{\text {qoc }}$ in this paper.

The action of quantum open-closed string field theory is then given by summing up the kinetic terms (4.3) and all the interaction terms (vertices) in Eq. (4.1),

$$
S_{\mathrm{qoc}}(\phi, \psi)=\sum_{g, b, n} \frac{1}{n ! b !\left|J_{1}\right| \cdots\left|J_{b}\right|} \mathcal{V}_{i_{1} \cdots i_{n} ; J_{1}, \ldots, J_{b}}^{g}\left(\phi^{J_{b}} \cdots \phi^{J_{1}}\right)\left(\psi^{i_{n}} \cdots \psi^{i_{1}}\right),
$$




$$
\mathcal{V}_{i_{1} \cdots i_{n} ; J_{1}, \ldots, J_{b}}^{g}:=\int_{\mathcal{V}_{b, m}^{g, n}}\langle\Omega|\left(\mathbf{e}_{c, i_{1}}, \ldots, \mathbf{e}_{c, i_{n}} ; \mathbf{e}_{o, J_{1}}, \ldots, \mathbf{e}_{o, J_{b}}\right),
$$

where $\mathbf{e}_{o, J}=\mathbf{e}_{o, j_{1}} \cdots \mathbf{e}_{o, j_{|J|}}$ for $J=\left(j_{1}, \ldots, j_{|J|}\right)$, and the summation $\Sigma_{g, b, n}$ is taken for all $g \geqslant 0, b \geqslant 0$ and $n \geqslant 0$ except for the cases $(g, b)=(0,0), n \leqslant 1,(g, n, b)=(0,0,1),\left|J_{1}\right| \leqslant 1$ and $(g, n, b)$ $=(1,0,0)$. In particular, the terms with $(g, n, b)=(0,2,0)$ and $(g, n, b)=(0,0,1)$ with $\left|J_{1}\right|=2$ are the kinetic terms of closed strings and open strings, respectively.

A quantum open-closed string field theory $S_{\text {qoc }}(\phi, \psi)$ is defined so that it satisfies the quantum master equation

$$
\frac{1}{2}\left(S_{\mathrm{qoc}}, S_{\mathrm{qoc}}\right)+\Delta S_{\mathrm{qoc}}=0 .
$$

Note that $\Delta S_{\text {qoc }}$ is the term peculiar to the quantum string field theory. $\Delta_{c}$ increases $g$ by one and $\Delta_{o}$ increases $b$ by one for $b>0$. The quantum master equation splits into separate equations for each genus $g$ and number of boundaries $b$. When we concentrate on the equations for $g=0$ and $b=0$ or 1 , we get

$$
\begin{gathered}
(g, b)=(0,0), \quad 0=\left(S_{S}, S_{S}\right)_{c}, \\
(g, b)=(0,1), \quad 0=\left(\tilde{S}_{D}, S_{S}\right)_{c}+\frac{1}{2}\left(\widetilde{S}_{D}, \widetilde{S}_{D}\right)_{o},
\end{gathered}
$$

where $S_{S}$ and $\widetilde{S}_{D}$ consist of the corresponding terms in $S_{\text {qoc }}$ in Eq. (4.5); explicitly, $S_{S}$ is of the same form as $S_{S}$ in Eq. (3.9) and $\widetilde{S}_{D}$ consists of $S_{D}$ in Eq. (3.9) with additional terms corresponding to $(k, l)=(k, 0)$ below,

$$
\begin{aligned}
& S_{S}(\psi)=\sum_{l \geqslant 2} \frac{1}{l !} \mathcal{V}_{i_{1} \cdots i_{l}} \psi^{i_{l} \cdots} \psi^{i_{1}} \in C(\psi),
\end{aligned}
$$

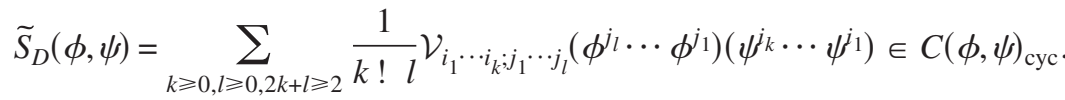

Here we dropped the index $g$ used in Eq. (4.5) since $g=0$. Namely, we denote

$$
\mathcal{V}_{i_{1} \cdots i_{l} ; \varnothing}^{g=0}=: \mathcal{V}_{i_{1} \cdots i_{l}}, \quad \mathcal{V}_{i_{1} \cdots i_{l}, J=\left(j_{1}, \ldots, j_{l}\right)}^{g=0}=: \mathcal{V}_{i_{1} \cdots i_{l} ; j_{1} \cdots j_{l}} .
$$

The action $S_{S}$ corresponds to punctured spheres [since the corresponding Riemann surfaces have no boundary $(\varnothing)$ ], whereas $\widetilde{S}_{D}$ corresponds to disks with punctures both in the disks and on the boundary of the disks. Equation (4.8) is often called a Maurer-Cartan equation.

A classical (tree) open-closed string field theory ${ }^{74}$ is then given by the action $S_{\text {toc }}(\phi, \psi)$ $=S_{S}(\psi)+\widetilde{S}_{D}(\phi, \psi)$ satisfying Eqs. (4.7) and (4.8), the Batalin-Vilkovisky ${ }^{3,4}$ classical open-closed master equations. The identity (4.7) implies that $S_{S}$ is just the action of the classical closed string field theory. ${ }^{73}$ Namely, $S_{S}$ has a cyclic $L_{\infty}$ structure. For the operadic construction of the classical closed string field theory, see Ref. 38. The relevant operad is the $L_{\infty}$ operad of nonplanar trees, where the composition of the trees corresponds to the twist-sewing of two $S^{1}$ s parametrizing two closed strings and/or boundaries in a Riemann surface picture. ${ }^{73,38}$

Just in the same way as for Eq. (3.10), one can define the following formal vector fields acting on $C(\phi, \psi)_{\text {cyc }}$ :

$$
\delta:=\delta_{S}+\delta_{D}, \quad \delta_{S}=\left(, S_{S}\right)_{c}, \quad \delta_{D}=\left(, \widetilde{S}_{D}\right)_{o} .
$$

The condition $(\delta)^{2}=0$ that $(\mathcal{H}, \mathfrak{l}, \mathfrak{n})$ is a cyclic OCHA is equivalent to the derivatives of the master equations (4.7) and (4.8), 


$$
\begin{gathered}
0=\left(,\left(S_{S}, S_{S}\right)_{c}\right)_{c}, \\
0=\left(,\left(\tilde{S}_{D}, S_{S}\right)_{c}+\frac{1}{2}\left(\tilde{S}_{D}, \tilde{S}_{D}\right)_{o}\right)_{o} .
\end{gathered}
$$

Here note that, as has been explained in Eq. (4.9), $\widetilde{S}_{D}$ consists of $S_{D}$ with the following additional terms:

$$
\frac{1}{l !} \mathcal{V}_{j_{1} \cdots j_{l} ;=\varnothing}\left(\psi^{j_{l}} \cdots \psi^{j_{1}}\right)
$$

each of which corresponds to a disk with punctures only in the bulk (the interior of the disk) and no punctures $J=\varnothing$ on the boundary. However, one can see that these terms drop out in Eq. (4.10): $\left(, \widetilde{S}_{D}\right)_{o}=\left(, S_{D}\right)_{o}$, since no open string field $\phi^{i}$ is included in Eq. (4.11). This is why we do not include the corresponding terms in the definition of (cyclic) OCHAs. Thus, a classical open-closed string field theory is a cyclic OCHA with the additional terms (4.11).

Of course, there exist situations in which these terms (4.11) are also important physically. For example, the terms (4.11) contribute to a constant term for open string field theory in discussing its deformation as in Sec. VI, and the constant term is relevant to D-brane mass, since the value of the action is believed to correspond to D-brane mass in open string field theory. But, it is enough to consider a cyclic OCHA structure in a classical open-closed string field theory at present if we examine its homotopy algebraic structures in the sense of the next section.

\section{MAURER-CARTAN EQUATION, MINIMAL MODEL, AND TREE OPEN-CLOSED STRING AMPLITUDES}

Homotopy algebras should have some homotopical properties. ${ }^{7,51}$ One of the key theorems in homotopy algebra is the minimal model theorem. The minimal model theorem for $A_{\infty}$ algebras was proved by Kadeishvili. ${ }^{30}$ For the construction of minimal models of $A_{\infty}$ structures, homological perturbation theory was developed by Refs. 17, 28, 18, 21, 19, and 20, for instance, and the form of a minimal model is then given explicitly in Refs. 54 and 41. Also, the existence of a stronger theorem, called the decomposition theorem in Refs. 33 and 36, is mentioned in Ref. 40 and proven by employing a kind of homological perturbation theory in Refs. 33 and 36 (see also Ref. 47). It is clear that the same arguments hold true for $L_{\infty}$ algebras, and in our previous paper ${ }^{35}$ we stated that they hold also for OCHAs.

In this section, we present the explicit form of a minimal model for an OCHA, which in the cyclic case can be thought of as the perturbative expansion of a classical open-closed string field theory.

Definition 5.1 (minimal open-closed homotopy algebra): An OCHA $\left(\mathcal{H}=\mathcal{H}_{c} \oplus \mathcal{H}_{o}, \mathfrak{l}, \mathfrak{n}\right.$ ) is called minimal if $l_{1}=0$ on $\mathcal{H}_{c}$ and $n_{0,1}=0$ on $\mathcal{H}_{o}$.

Theorem 5.2 (Minimal model theorem for open-closed homotopy algebras ${ }^{35}$ ): For any OCHA, there exists a minimal OCHA and an OCHA quasi-isomorphism from the minimal OCHA to the original OCHA.

The minimal model theorem holds also for COCHAs. Namely, for any COCHA, there exists a minimal COCHA and a COCHA quasi-isomorphism from the minimal COCHA to the original COCHA. This fact also follows from the explicit minimal model we shall construct here.

First of all, we fix a Hodge decomposition of the complex $\left(\mathcal{H}, d=l_{1}+n_{0,1}\right)$. Namely, for $d_{c}$ $=l_{1}$ and $d_{o}=n_{0,1}$, we give Hodge decompositions of the complexes $\left(\mathcal{H}_{c}, d_{c}\right)$ and $\left(\mathcal{H}_{o}, d_{o}\right)$ separately, by fixing degree minus one (homotopy) operators $h_{c}: \mathcal{H}_{c} \rightarrow \mathcal{H}_{c}$ and $h_{o}: \mathcal{H}_{o} \rightarrow \mathcal{H}_{o}$,

$$
d_{c} h_{c}+h_{c} d_{c}+\iota_{c} \circ \pi_{c}=\mathbf{1}_{c}, \quad d_{o} h_{o}+h_{o} d_{o}+\iota_{o} \circ \pi_{o}=\mathbf{1}_{o} .
$$

Here, $\pi$ and $\iota$ indicate the projection to and the inclusion into the corresponding cohomologies. Thus, these data give a contraction (deformation retract) of $\mathcal{H}=\mathcal{H}_{c} \oplus \mathcal{H}_{o}$ as a graded vector space (see Ref. 35), 


$$
(H(\mathcal{H}) \underset{\pi}{\stackrel{\iota}{\rightleftarrows}} \mathcal{H}, h),
$$

where $\iota:=\iota_{c}+\iota_{o}, \pi:=\pi_{c}+\pi_{o}$ and $h=h_{c}+h_{o}$.

We would like to follow the arguments in Refs. 31 and 33, where a minimal model is obtained by a process of solving the Maurer-Cartan equation for an $A_{\infty}$ algebra. For OCHAs, the MaurerCartan equations are defined as follows. ${ }^{35}$

Definition 5.3 (Maurer-Cartan equation): For an OCHA $(\mathcal{H}, \mathfrak{l}, \mathfrak{n})$ and degree zero elements $\bar{c} \in \mathcal{H}_{c}$ and $\bar{o} \in \mathcal{H}_{o}$, we call the following pair of equations

$$
0=\sum_{k} \frac{1}{k !} l_{k}(\bar{c}, \ldots, \bar{c}), \quad 0=\sum_{k, l} \frac{1}{k !} n_{k, l}(\bar{c}, \ldots, \bar{c} ; \bar{o}, \ldots, \bar{o})
$$

the Maurer-Cartan equations for the OCHA $(\mathcal{H}, \mathfrak{l}, \mathfrak{n})$

Remark 5.4: Recall that, for the cyclic $A_{\infty}$ or $L_{\infty}$ case, the Maurer-Cartan equations are just the equations of motions for the action (of the corresponding string field theory). ${ }^{31,33}$ In field theory, the equations of motions are defined by the derivatives of the action with respect to the fields. For instance, for classical closed string field theory with the action $S_{S}$, the equations of motions are $0=\left(\vec{\partial} / \partial \psi^{i}\right) S_{S}$ for each $i$. Here, since the BV bracket $(,)_{c}:=\left(\overleftarrow{\partial} / \partial \psi^{i}\right) \omega_{c}^{i j}\left(\vec{\partial} / \partial \psi^{j}\right)$ is nondegenerate, the equations of motions are equivalent to $0=\left(, S_{S}\right)\left(=\delta_{S}\right)$. Usually, we set degree nonzero fields to be zero and concentrate on the solutions for the fields of degree zero. Then, further identifying $\vec{\partial} / \partial \psi^{i}$ with $\mathbf{e}_{c}^{i}$ in $0=\delta_{S}$, one obtains the Maurer-Cartan equations for the $L_{\infty}$ algebra, which is the first equation in Eq. (5.3). However, note that, for a $\operatorname{COCHA}(\mathcal{H}, \omega, \mathfrak{l}, \mathfrak{n})$, the zeroes of the corresponding odd formal vector field $\delta=\delta_{S}+\delta_{D}$ are not the same as solutions to the equations of motions $0=(, S)=(, S)_{c}+(, S)_{o}$, or separately

$$
0=\frac{\overleftarrow{\partial}}{\partial \psi^{j}} \omega_{c}^{i j} \frac{\vec{\partial}}{\partial \psi^{j}}\left(S_{S}+S_{D}\right)=0, \quad 0=\frac{\overleftarrow{\partial}}{\partial \phi^{i}} \omega_{o}^{i j} \frac{\vec{\partial}}{\partial \phi^{j}} S_{D}=0
$$

for the COCHA. Namely, the first equation above includes the term $\left(, S_{D}\right)_{c}$, the corresponding term of which is absent in the Maurer-Cartan equations (5.3) for the COCHA.

One can see that, if one solves the equations of motions, instead of solving the Maurer-Cartan equations, the resulting structure includes terms corresponding to $b>1$.

If we apply the arguments in Refs. 31 and 33 to an OCHA, the Maurer-Cartan equations for an OCHA should be considered formally for the pair of string fields $(\Psi, \Phi) \in\left(\mathcal{H}_{c} \otimes \mathcal{H}_{c}^{*}, \mathcal{H}_{o}\right.$ $\left.\otimes \mathcal{H}_{o}^{*}\right)$ instead of their degree zero parts $(\bar{c}, \bar{o}) \in\left(\mathcal{H}_{c}^{0}, \mathcal{H}_{o}^{0}\right)$.

Then, for instance for the $L_{\infty}$ part, solving the Maurer-Cartan equation recursively one gets first an $L_{\infty}$ quasi-isomorphism $\left\{f_{k}\right\}_{k \geqslant 1}:\left(H\left(\mathcal{H}_{c}\right)\right)^{\otimes k} \rightarrow \mathcal{H}_{c}$. This somewhat physical procedure is closely related to the homological perturbation theory developed earlier, and in particular, $f:=$ $\oplus_{k} f_{k} \in \oplus_{k} \operatorname{Hom}\left(\left(H\left(\mathcal{H}_{c}\right)\right)^{\otimes k}, \mathcal{H}_{c}\right)$ is just what is called a twisting cochain $\tau$ (see Ref. 29 for the DGLA case). Then, substituting $\mathfrak{f}$ instead of $\Psi$ into the initial Maurer-Cartan equation, one obtains an equation on $H\left(\mathcal{H}_{c}\right)$, which is in fact the Maurer-Cartan equation for the corresponding minimal $L_{\infty}$ algebra, so one can read the minimal $L_{\infty}$ structure from the Maurer-Cartan equation. For the case of an OCHA, its minimal model is obtained by first considering the Maurer-Cartan equation for the $L_{\infty}$ algebra as above and, after that, considering the Maurer-Cartan equation for $\mathfrak{n}$.

For an $L_{\infty}$ algebra $\left(\mathcal{H}_{c}, \mathfrak{l}\right)$, a minimal $L_{\infty}$ algebra and an $L_{\infty}$ quasi-isomorphism $\left\{f_{l}\right\}_{l \geqslant 1}:\left(H\left(\mathcal{H}_{c}\right), \mathfrak{l}^{\prime}\right) \rightarrow\left(\mathcal{H}_{c}, \mathfrak{l}\right)$ are constructed as follows.

We set $f_{1}=\iota_{c}: H\left(\mathcal{H}_{c}\right) \rightarrow \mathcal{H}_{c}$, and assume that we have $\left\{f_{l}:\left(H\left(\mathcal{H}_{c}\right)\right)^{\otimes l} \rightarrow \mathcal{H}_{c}\right\}_{l \geqslant 1}$ for $l \leqslant n-1$. Then, for $c_{1}^{\prime}, \ldots, c_{n}^{\prime} \in H\left(\mathcal{H}_{c}\right), f_{n}$ is defined by

$$
f_{n}\left(c_{1}^{\prime}, \ldots, c_{n}^{\prime}\right)=-h_{c} \sum_{k_{1}+\cdots+k_{j}=n} \sum_{\sigma \in \mathfrak{S}_{n}} \frac{(-1)^{\epsilon(\sigma)}}{k_{1} ! k_{2} ! \cdots k_{j} ! \cdot j !} l_{j}\left(f_{k_{1}} \otimes f_{k_{2}} \otimes \cdots \otimes f_{k_{j}}\right)\left(c_{\sigma(1)}^{\prime}, \ldots, c_{\sigma(n)}^{\prime}\right) .
$$

The minimal $L_{\infty}$ structure is then determined by 


$$
l_{n}^{\prime}\left(c_{1}^{\prime}, \ldots, c_{n}^{\prime}\right)=\pi_{c} \sum_{k_{1}+\cdots+k_{j}=n} \sum_{\sigma \in \mathfrak{S}_{n}} \frac{(-1)^{\epsilon(\sigma)}}{k_{1} ! k_{2} ! \cdots k_{j} ! \cdot j !} l_{j}\left(f_{k_{1}} \otimes f_{k_{2}} \otimes \cdots \otimes f_{k_{j}}\right)\left(c_{\sigma(1)}^{\prime}, \ldots, c_{\sigma(n)}^{\prime}\right),
$$

in particular, for $l=2$ one gets $l_{2}^{\prime}=H\left(l_{2}\right):=\pi_{c} \circ l_{2} \circ\left(\iota_{c}\right)^{\otimes 2}$.

Once the $L_{\infty}$ quasi-isomorphism $\left\{f_{l}\right\}_{l \geqslant 1}$ is given, we have an OCHA $\left(H\left(\mathcal{H}_{c}\right) \oplus \mathcal{H}_{o}, \mathfrak{l}^{\prime}, \mathfrak{n}^{\prime \prime}\right)$ for some $\mathfrak{n}^{\prime \prime}$. Next we should construct $\left\{f_{k, l}:\left(H\left(\mathcal{H}_{c}\right)\right)^{\otimes k} \otimes\left(H\left(\mathcal{H}_{o}\right)\right)^{\otimes l} \rightarrow H\left(\mathcal{H}_{o}\right)\right\}_{k+l \geqslant 1}$ and $\mathfrak{n}^{\prime}$ $=\left\{n_{k, l}^{\prime}:\left(H\left(\mathcal{H}_{c}\right)\right)^{\otimes k} \otimes\left(H\left(\mathcal{H}_{o}\right)\right)^{\otimes l} \rightarrow H\left(\mathcal{H}_{o}\right)\right\}_{2 k+l \geqslant 2}$; these are obtained in a similar way as follows. $f_{0,1}$ is given as inclusion $f_{0,1}=\iota_{o}: H\left(\mathcal{H}_{o}\right) \rightarrow \mathcal{H}_{o} . f_{r, s}$ and $f_{p, q}$ are ordered as $f_{r, s}<f_{p, q}$ if $r+s<p+q$ or $r<p$ for $r+s=p+q$. Then, a similar recursive procedure as above can be carried out also here. For $c_{1}^{\prime}, \ldots, c_{n}^{\prime} \in H\left(\mathcal{H}_{c}\right)$ and $o_{1}^{\prime}, \ldots, o_{m}^{\prime} \in H\left(\mathcal{H}_{o}\right), f_{n, m}$ is determined by

$$
\begin{aligned}
& f_{n, m}\left(c_{1}^{\prime}, \ldots, c_{n}^{\prime} ; o_{1}^{\prime}, \ldots, o_{m}^{\prime}\right) \\
& =-h_{o} \sum \frac{(-1)^{\epsilon(\sigma)}}{i !\left(r_{1} ! \cdots r_{i} !\right)\left(p_{1} ! \cdots p_{j} !\right)} n_{i, j}\left(f_{r_{1}} \otimes \cdots \otimes f_{r_{i}} \otimes f_{p_{1}, q_{1}} \otimes \cdots \otimes f_{p_{j}, q_{j}}\right) \\
& \quad\left(c_{\sigma(1)}^{\prime}, \ldots, c_{\sigma(n)}^{\prime} ; o_{1}^{\prime}, \ldots, o_{m}^{\prime}\right),
\end{aligned}
$$

where the summation $\Sigma$ runs over all $r_{1}, \ldots, r_{i}, p_{1}, \ldots, p_{j}, q_{1}, \ldots, q_{j}$ such that $\left(r_{1}+\cdots+r_{i}\right)+\left(p_{1}\right.$ $\left.+\cdots+p_{j}\right)=n,\left(q_{1}+\cdots+q_{j}\right)=m$, and also all $\sigma \in \mathfrak{S}_{n}$.

Then $\mathfrak{n}^{\prime}=\left\{n_{k, l}^{\prime}\right\}_{2 k+l \geqslant 2}$ is obtained by replacing $-h_{o}$ above with $\pi_{o}$,

$$
\begin{aligned}
& n_{n, m}^{\prime}\left(c_{1}^{\prime}, \ldots, c_{n}^{\prime} ; o_{1}^{\prime}, \ldots, o_{m}^{\prime}\right) \\
& \quad=\pi_{o} \sum \frac{(-1)^{\epsilon(\sigma)}}{i !\left(r_{1} ! \cdots r_{i} !\right)\left(p_{1} ! \cdots p_{j} !\right)} n_{i, j}\left(f_{r_{1}} \otimes \cdots \otimes f_{r_{i}} \otimes f_{p_{1}, q_{1}} \otimes \cdots \otimes f_{p_{j}, q_{j}}\right) \\
& \quad\left(c_{\sigma(1)}^{\prime}, \ldots, c_{\sigma(n)}^{\prime} ; o_{1}^{\prime}, \ldots, o_{m}^{\prime}\right),
\end{aligned}
$$

where the summation $\sum$ stands for the same one as above. In particular, for $2 k+l=2$ one gets $n_{0,2}^{\prime}=H\left(n_{0,2}\right):=\pi_{o} \circ n_{0,2}{ }^{\circ}\left(\iota_{o}\right)^{\otimes 2}$ and $n_{1,0}^{\prime}=H\left(n_{1,0}\right):=\pi_{o}{ }^{\circ} n_{1,0}{ }^{\circ} \iota_{c}$. In the equation above, we used the convention presented in Definition 2.10.

For a COCHA $(\mathcal{H}, \omega, \mathfrak{l}, \mathfrak{n})$, we do this construction by starting with an orthogonal Hodge decomposition with respect to the symplectic form $\omega$. Namely, we give a decomposition

$$
(H(\mathcal{H}) \underset{\pi}{\stackrel{\iota}{\rightleftarrows}} \mathcal{H}, h)
$$

of $\mathcal{H}$ in Eq. (5.2) with a homotopy $h: \mathcal{H} \rightarrow \mathcal{H}$ satisfying $\omega(\mathbf{1} \otimes h)=\omega(h \otimes \mathbf{1})$, where $\mathbf{1}:=\mathbf{1}_{c} \oplus \mathbf{1}_{o}$. The existence of such a homotopy $h$ follows from the nondegeneracy of $\omega$ and the cyclicity for the terms $\omega_{c}\left(l_{1} \otimes \mathbf{1}_{c}\right)$ and $\omega_{o}\left(n_{0,1} \otimes \mathbf{1}_{o}\right)$, and then $\omega\left(\mathbf{1} \otimes\left(\iota^{\circ} \pi\right)\right)=\omega\left(\left(\iota^{\circ} \pi\right) \otimes \mathbf{1}\right)$ also holds. Then, for the COCHA $(\mathcal{H}, \omega, \mathfrak{l}, \mathfrak{n})$, forgetting the cyclic structure $\omega$ having already used it to fix the contraction (5.2), one can obtain a minimal model $\left(H(\mathcal{H}), \mathfrak{l}^{\prime}, \mathfrak{n}^{\prime}\right)$ as an OCHA by the construction we have seen above. The resulting minimal OCHA $\left(H(\mathcal{H}), \mathfrak{l}^{\prime}, \mathfrak{n}^{\prime}\right)$ is in fact cyclic with respect to the induced inner product $\omega^{\prime}:=\omega(\iota \otimes \iota)$ and the OCHA quasi-isomorphism a COCHA quasiisomorphism.

To summarize we give the following.

Theorem 5.5: $\left(H(\mathcal{H}), \mathfrak{l}^{\prime}, \mathfrak{n}^{\prime}\right)$ forms a minimal OCHA and $\mathfrak{f}:=\left\{\left\{f_{l}\right\}_{l \geqslant 1},\left\{f_{k, l}\right\}_{k+l \geqslant 1}\right\}$ is an OCHA quasi-isomorphism $\mathfrak{f}:\left(H(\mathcal{H}), \mathfrak{l}^{\prime}, \mathfrak{n}^{\prime}\right) \rightarrow(\mathcal{H}, \mathfrak{l}, \mathfrak{n})$.

Theorem 5.6: For a COCHA $(\mathcal{H}, \omega, \mathfrak{l}, \mathfrak{n})$ and an orthogonal Hodge decomposition with respect to $\omega,\left(H(\mathcal{H}), \omega^{\prime}, \mathfrak{l}^{\prime}, \mathfrak{n}^{\prime}\right)$ forms a minimal COCHA and $\mathfrak{f}:=\left\{\left\{f_{l}\right\}_{l \geqslant 1},\left\{f_{k, l}\right\}_{k+l \geqslant 1}\right\}$ is a COCHA quasi-isomorphism $\mathfrak{f}:\left(H(\mathcal{H}), \omega^{\prime}, \mathfrak{l}^{\prime}, \mathfrak{n}^{\prime}\right) \rightarrow(\mathcal{H}, \omega, \mathfrak{l}, \mathfrak{n})$.

Since the explicit forms are given, one can check the cyclicity directly in a similar way to that in the $A_{\infty}$ case (see Ref. 33).

Remark 5.7 (rooted planar tree graphs): One can also present an alternate description of this 


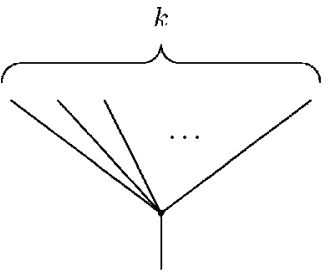

(a)

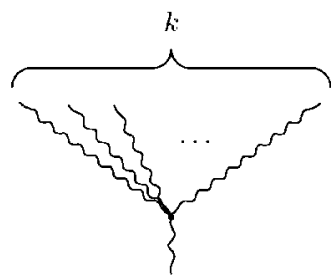

(b)

FIG. 1. (a) The $k$-corolla, the planar tree corresponding to the $A_{\infty}$ structure $m_{k}=n_{0, k}$. (b) The $k$-corolla, the nonplanar tree corresponding to the $L_{\infty}$ structure $l_{k}$.

minimal model in terms of rooted planar trees in a similar way as for $A_{\infty}$ algebras (see Refs. 41, $31,33,52,7,11,15,16,27,36,47,51$, and 60 , etc.). This is related to Feynman graphs in field theory. For an $A_{\infty}$ algebra $\left(\mathcal{H}_{o},\left\{n_{0, k}\right\}\right)$, it is convenient to associate $n_{0, k}$ to the $k$-corolla of planar rooted trees. An $L_{\infty}$ algebra also has such a description, where the $L_{\infty}$ structure $l_{k}$ is associated with the $k$-corolla of nonplanar rooted trees (Fig. 1). In our OCHA, we need to introduce also $n_{k, l}$, to which we associate a mixed corolla as in Fig. 2. As stated previously, from a string theory viewpoint, $l_{k}$ corresponds to the sphere with $(k+1)$-(closed string) punctures and $n_{k, l}$ corresponds to the disk with $(k+1)$-(open string) punctures on the boundary and $l$-(closed string) punctures in the bulk (interior) of the disk. In fact, one may think of the tubular neighborhood of these tree graphs as the corresponding world sheet, where we take strips and cylinders for the neighborhood of the straight lines and meandering lines, respectively. The minimal OCHA structure $l_{k}^{\prime}$ and $n_{k, l}^{\prime}$ are then obtained by grafting corollas in all possible ways such that straight lines are grafted to straight and wiggly to wiggly (Fig. 3), where we assign to corollas the corresponding multilinear maps $l_{k}, n_{p, q}$, and to internal edges $h_{c}, h_{o}$, and so on. Physically, $h_{c}$ and $h_{o}$ are the propagators for closed string and open string, respectively.

Remark 5.8 (string amplitude): For a classical open-closed string field theory $S=S_{S}+S_{D}$, the string amplitudes are obtained as follows. Let $(\mathcal{H}, \omega, \mathfrak{l}, \mathfrak{n})$ be the corresponding COCHA, and suppose that its minimal COCHA $\left(H(\mathcal{H}), \omega^{\prime}, \mathfrak{l}^{\prime}, \mathfrak{n}^{\prime}\right)$ is constructed as above.

By definition, string field theory is constructed so that its perturbative expansion reproduces the corresponding world sheet string amplitudes. Thus,

$$
\mathcal{V}_{k+1}^{\prime}:=\omega_{c}^{\prime}\left(l_{k}^{\prime} \otimes \mathbf{1}_{c}\right), \quad \mathcal{V}_{k, l+1}^{\prime}=\omega_{o}^{\prime}\left(n_{k, l}^{\prime} \otimes \mathbf{1}_{o}\right)
$$

just define the on-shell $(k+1)$-closed strings sphere amplitudes and $k$-closed $(l+1)$-open string disk amplitudes, respectively. Moreover, the $n$-closed string disk amplitude, which we denote $\mathcal{V}_{n, 0}^{\prime}$, is given by composing the $L_{\infty}$ quasi-isomorphism with $\mathcal{V}_{k, 0}$ as follows:

$$
\mathcal{V}_{n, 0}^{\prime}=\sum_{i=1}^{n} \sum_{k_{1}+\cdots+k_{i}=n} \frac{1}{k_{1} ! \cdots k_{i} !} \mathcal{V}_{i, 0}\left(f_{k_{1}} \otimes \cdots \otimes f_{k_{i}}\right)
$$

for $\mathfrak{f}=\left\{f_{k}\right\}_{k \geqslant 1}$, the $L_{\infty}$ quasi-isomorphism.

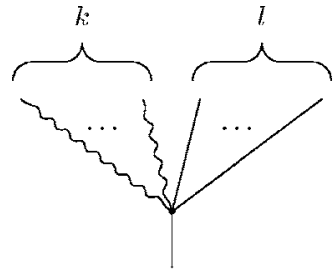

(a)

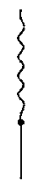

(b)

FIG. 2. (a) The $(k, l)$-corolla corresponding to $n_{k, l}$ (b) For the open-closed case, the $(k, l)$-corollas include the $(1,0)$-corolla corresponding to $n_{1,0}$. 


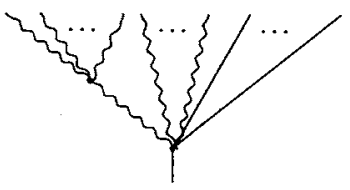

(a)

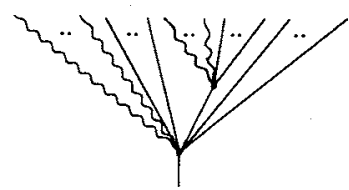

(b)

FIG. 3. Grafting of corollas. (a) Wiggly to wiggly. (b) Straight to straight.

Note also that we can prove the decomposition theorem for a (cyclic) OCHA,${ }^{35}$ which implies that all classical open-closed string field theories constructed on a fixed conformal background (the data of the conformal field theory on the Riemann surfaces) are isomorphic. From the field theory viewpoint, an OCHA morphism is a field transformation and in particular an OCHA isomorphism is a field redefinition. Since field theory actions related by a field redefinition are physically equivalent, one can say the decomposition theorem can prove the equivalence of all classical open-closed string field theories constructed on a fixed conformal background. (See Ref. 33 for the $A_{\infty}$ case.)

\section{DEFORMATION OF $\boldsymbol{A}_{\infty}$ STRUCTURES AND THE FORMALITY THEOREM}

For an $L_{\infty}$ algebra $\left(\mathcal{H}_{c}, \mathfrak{l}\right)$ and an $A_{\infty}$ algebra $\left(\mathcal{H}_{o}, \mathfrak{m}\right)$, if there exists an OCHA $\left(\mathcal{H}=\mathcal{H}_{c}\right.$ $\left.\oplus \mathcal{H}_{o}, \mathfrak{l}, \mathfrak{n}\right)$ whose subalgebra $\left(\mathcal{H}_{o},\left\{n_{0, k}\right\}_{k \geqslant 1}\right)$ coincides with $\left(\mathcal{H}_{o},\left\{m_{k}\right\}_{k \geqslant 1}\right)$, one obtains deformations of the $A_{\infty}$ algebra $\left(\mathcal{H}_{o},\left\{m_{k}\right\}_{k \geqslant 1}\right)$ parametrized by the $L_{\infty}$ algebra $\left(\mathcal{H}_{c}, \mathfrak{l}\right)$. On the other hand, the whole deformation space of the $A_{\infty}$ algebra is also described by a moduli space of the $L_{\infty}$ algebra $\left.\left(\operatorname{Coder}\left(T^{c} \mathcal{H}_{o}\right), \mathfrak{m},[],\right)\right)$, which we denote by $\left(\mathcal{H}_{c}^{\prime}, \mathfrak{l}^{\prime}\right)$. The maps $\left\{n_{k, l}\right\}$ for $k \geqslant 1$ define an $L_{\infty}$ morphism from $\left(\mathcal{H}_{c}, \mathfrak{l}\right)$ to $\left(\mathcal{H}_{c}^{\prime}, \mathfrak{l}^{\prime}\right)$. The defining equation for an OCHA (2.6) just converts to the defining equation for an $L_{\infty}$ morphism (2.5). ${ }^{35}$ In Sec. VI A we reexplain this in a more explicit way. Such structure appears in various aspects of mathematical physics; the relation to deformation quantization by Kontsevich ${ }^{40}$ is explained in Sec. VI B, and the application to the open-closed topological B-model ${ }^{26}$ from the viewpoint of the homological mirror symmetry setup ${ }^{39}$ is discussed in Sec. VI C. Note also that, in string field theory, this picture is related to the arguments in Sec. 8 of Ref. 74.

\section{A. Deformations of $\boldsymbol{A}_{\infty}$ structures from open-closed homotopy algebras}

Definition 6.1 [(graded) Gerstenhaber bracket ${ }^{12}$ ]: For $\mathcal{H}_{o}$ a Z-graded vector space, let $\operatorname{Hom}_{k}^{r}$ $:=\operatorname{Hom}^{r}\left(\mathcal{H}_{o}^{\otimes k}, \mathcal{H}_{o}\right)$ be the space of degree $r k$-linear maps, and

$$
\text { Hom }:=\oplus_{r} \operatorname{Hom}^{r}, \quad \operatorname{Hom}^{r}:=\oplus_{k \geqslant 0} \operatorname{Hom}_{k}^{r} .
$$

It is known that Hom is in one-to-one correspondence with the space of coderivations on $T^{c}\left(\mathcal{H}_{o}\right)$, $\operatorname{Coder}\left(T^{c}\left(\mathcal{H}_{o}\right)\right) .{ }^{65}$ For two elements in $\operatorname{Coder}\left(T^{c}\left(\mathcal{H}_{o}\right)\right)$, the commutator of any two elements in fact belongs to $\operatorname{Coder}\left(T^{c}\left(\mathcal{H}_{o}\right)\right)$, and further satisfies the Jacobi identity. After a shift in degree, this induces a graded Lie bracket on Hom, which is the Gerstenhaber bracket. For $m \in \operatorname{Hom}_{k}^{r}, m^{\prime}$ $\in \operatorname{Hom}_{k^{\prime}}^{r^{\prime}}$ and $o_{1}, \ldots, o_{k+k^{\prime}-1} \in \mathcal{H}_{o}$, the graded Lie bracket $\left[m, m^{\prime}\right] \in \operatorname{Hom}_{k+k^{\prime}-1}^{r+r^{\prime}}$ is defined by

$$
\begin{gathered}
{\left[m, m^{\prime}\right]=m \circ m^{\prime}-(-1)^{r \cdot r^{\prime}} m^{\prime} \circ m,} \\
m \circ m^{\prime}\left(o_{1}, \ldots, o_{k+k^{\prime}-1}\right)=\sum_{i=0}^{k-1}(-1)^{l^{\prime}\left(o_{1}+\cdots+o_{i}\right)} m\left(o_{1}, \ldots, o_{i}, m^{\prime}\left(o_{i+1}, \ldots, o_{i+k^{\prime}}\right), o_{i+k^{\prime}+1}, \ldots, o_{k+k^{\prime}-1}\right) .
\end{gathered}
$$

Thus, (Hom, $[$,$] ) forms a graded Lie algebra. { }^{12,65}$

In the supermanifold description in Sec. III, this Gerstenhaber bracket corresponds to the graded Lie bracket of formal vector fields on the corresponding formal supermanifold. 
Furthermore, let us denote by $\bar{m} \in \mathrm{Hom}^{1}$ the degree one element corresponding to a degree one coderivation in $\left(\mathcal{H}_{c}^{\prime}, \mathfrak{l}^{\prime}\right)=\operatorname{Coder}\left(T\left(\mathcal{H}_{o}\right)\right)$, that is, an $A_{\infty}$ structure on $\mathcal{H}_{o}$. Then, it is clear that $[\bar{m}, \bar{m}]=0$ holds and (Hom, $d,[$,$] ) forms a DGLA with d=[\bar{m}$,$] .$

Remark 6.2 (suspension): A DGLA is described as an $L_{\infty}$ algebra through the suspension $s$. For the DGLA (Hom, $d,[]$,$) , the suspension is a degree shifting operator$

$$
s: \operatorname{Hom} \rightarrow \operatorname{Hom}[1]=: \mathcal{H}_{c}^{\prime} .
$$

By this, a degree $r$ element $m \in \operatorname{Hom}^{r}$ is mapped to be a degree $(r-1)$ element $s(m)$ $\in \operatorname{Hom}[1]^{r-1}=\mathcal{H}_{c}^{\prime r-1}$. This actually converts the degree preserving bracket $[]:$, Hom $\otimes$ Hom $\rightarrow$ Hom into a degree one bilinear map $l_{2}^{\prime}: \mathcal{H}_{c}^{\prime} \otimes \mathcal{H}_{c}^{\prime} \rightarrow \mathcal{H}_{c}^{\prime}$ defined by

$$
l_{2}^{\prime}\left(s(m), s\left(m^{\prime}\right)\right):=s\left((-1)^{r}\left[m, m^{\prime}\right]\right), \quad m \in \operatorname{Hom}^{r}, \quad m^{\prime} \in \operatorname{Hom}^{r^{\prime}} .
$$

One can see that the graded anticommutativity $\left[m^{\prime}, m\right]=-(-1)^{r r^{\prime}}\left[m, m^{\prime}\right]$ is in fact replaced by the graded commutativity in Eq. $(2.3), l_{2}^{\prime}\left(s\left(m^{\prime}\right), s(m)\right)=(-1)^{(r-1)\left(r^{\prime}-1\right)} l_{2}^{\prime}\left(s(m), s\left(m^{\prime}\right)\right)$, where $l_{1}^{\prime}$ is given simply by $l_{1}^{\prime}=s \mathrm{~d} s^{-1}=d$. Then $\left(\mathcal{H}_{c}^{\prime}, \mathfrak{l}^{\prime}=\left\{l_{1}^{\prime}, l_{2}^{\prime}, l_{3}^{\prime}=l_{4}^{\prime}=\cdots=0\right\}\right)$ forms an $L_{\infty}$ algebra.

Now, let us express the open-closed multilinear maps as

$$
n_{k, l}\left(c_{1}, \ldots, c_{k} ; o_{1}, \ldots, o_{l}\right)=:\left(n_{l}^{*}\left(c_{1}, \ldots, c_{k}\right)\right)\left(o_{1}, \ldots, o_{l}\right),
$$

where $n_{l}^{*}\left(c_{1}, \ldots, c_{k}\right)$ belongs to $\operatorname{Hom}_{k}^{\operatorname{deg}\left(c_{1}\right)+\cdots+\operatorname{deg}\left(c_{k}\right)+1}$. By this, the second term of the OCHA relation (2.6) can be rewritten as

$$
-(-1)^{\left(c_{\sigma(1)}+\cdots+c_{\sigma(l)}+1\right)} \frac{1}{2}\left[n_{m+1-k}^{*}\left(c_{\sigma(1)}, \ldots, c_{\sigma(l)}\right), n_{k}^{*}\left(c_{\sigma(l+1)}, \ldots, c_{\sigma(n)}\right)\right]\left(o_{1}, \ldots, o_{m}\right),
$$

which, acting further with the suspension $s$ on the equation above, yields

$$
-\frac{1}{2} l_{2}^{\prime}\left(s\left(n_{m+1-k}^{*}\right)\left(c_{\sigma(1)}, \ldots, c_{\sigma(l)}\right), s\left(n_{k}^{*}\right)\left(c_{\sigma(l+1)}, \ldots, c_{\sigma(n)}\right)\right)\left(o_{1}, \ldots, o_{m}\right) .
$$

Thus, one obtains

$$
\begin{aligned}
& \sum_{k, l \geqslant 0} \sum_{p=0}^{m-k} \sum_{\sigma \in \mathfrak{S}_{n}} \frac{1}{2} \frac{(-1)^{\epsilon(\sigma)}}{l !(n-l) !} l_{2}^{\prime}\left(s\left(n_{m+1-k}^{*}\right)\left(c_{\sigma(1)}, \ldots, c_{\sigma(l)}\right), s\left(n_{k}^{*}\right)\left(c_{\sigma(l+1)}, \ldots, c_{\sigma(n)}\right)\right) \\
& \quad=\sum_{\sigma \in \mathfrak{S}_{n}} \sum_{l=1}^{n} \frac{(-1)^{\epsilon(\sigma)}}{l !(n-l) !} s\left(n_{m}^{*}\right)\left(l_{l}\left(c_{\sigma(1)}, \ldots, c_{\sigma(l)}\right), c_{\sigma(l+1)} \ldots, c_{\sigma(n)}\right) .
\end{aligned}
$$

These are just the defining equations for an $L_{\infty}$ morphism (2.5). By treating the $l=0$ and $l=n$ cases separately in the first line of the equation above, it becomes just the condition that the collection of multilinear maps $s\left(n_{k}^{*}\right):\left(\mathcal{H}_{c}\right)^{\otimes *} \rightarrow \operatorname{Hom}_{k}[1]$ forms an $L_{\infty}$ morphism from $\left(\mathcal{H}_{c}, \mathfrak{l}\right)$ to $\left(\mathcal{H}_{c}^{\prime}, \mathfrak{l}^{\prime}\right)$. Here, note that $l_{1}^{\prime}=[\bar{m}$,$] and \bar{m}=\Sigma_{l \geqslant 0} m_{k}$, the $A_{\infty}$ structure included in $(\mathcal{H}, \mathfrak{l}, \mathfrak{n})$ as $m_{l}:=n_{0, l}$. From these arguments, it is clear that the converse also holds.

Theorem 6.3 (Ref. 35): For an OCHA $(\mathcal{H}, \mathfrak{l}, \mathfrak{n})$, let $\left(\mathcal{H}_{c}^{\prime}, \mathfrak{l}^{\prime}\right)$ denote the DGLA Coder $\left(T^{c} \mathcal{H}_{o}\right)$. The OCHA structure gives an $L_{\infty}$ morphism from $\left(\mathcal{H}_{c}, \mathfrak{l}\right)$ to $\left(\mathcal{H}_{c}^{\prime}, \mathfrak{l}^{\prime}\right)$. Conversely, if there exists an $L_{\infty}$ algebra $\left(\mathcal{H}_{c}, \mathfrak{l}\right)$ and an $L_{\infty}$ morphism from it to the DGLA $\left(\mathcal{H}_{c}^{\prime}, \mathfrak{l}^{\prime}\right)$ of an $A_{\infty}$ algebra $\left(\mathcal{H}_{o}, \mathfrak{m}\right)$, then one obtains an OCHA.

For an $L_{\infty}$ algebra $\left(\mathcal{H}_{c}, \mathfrak{l}\right)$, let us denote by $\mathcal{M C}\left(\mathcal{H}_{c}, \mathfrak{l}\right)$ the solution space of the Maurer-Cartan equation

$$
\mathcal{M C}\left(\mathcal{H}_{c}, \mathfrak{l}\right)=\left\{\bar{c} \in \mathcal{H}_{c}^{0} \mid 0=\sum_{k \geqslant 1} \frac{1}{k !} l_{k}(\bar{c}, \ldots, \bar{c})\right\},
$$

where $\mathcal{H}_{c}^{0}$ is the degree zero subvector space of $\mathcal{H}_{c}$. In addition, we have an equivalence relation $\sim$ called gauge equivalence between the solutions of the Maurer-Cartan equation. The moduli 
space of the solution space of the Maurer-Cartan equation for $\left(\mathcal{H}_{c}, \mathfrak{l}\right)$ is defined by

$$
\mathcal{M}\left(\mathcal{H}_{c}, \mathfrak{l}\right)=\mathcal{M C}\left(\mathcal{H}_{c}, \mathfrak{l}\right) / \sim
$$

Suppose that we have an $L_{\infty}$ morphism $\mathfrak{f}:\left(\mathcal{H}_{c}, \mathfrak{l}\right) \rightarrow\left(\mathcal{H}_{c}^{\prime}, \mathfrak{l}^{\prime}\right)$ between two $L_{\infty}$ algebras. Then it is known that the $L_{\infty}$ morphism $\mathfrak{f}$ induces a map $\mathfrak{f}: \mathcal{M}\left(\mathcal{H}_{c}, \mathfrak{l}\right) \rightarrow \mathcal{M}\left(\mathcal{H}_{c}^{\prime}, \mathfrak{l}^{\prime}\right)$, and furthermore it is an isomorphism if $\mathfrak{f}$ is an $L_{\infty}$ quasi-isomorphism (cf. Ref. 40). Similar facts hold also for $A_{\infty}$ algebras and also for OCHAs, ${ }^{35}$ but what is relevant here is just the $L_{\infty}$ case.

Note that, for an $L_{\infty}$ algebra (DGLA) $\left(\mathcal{H}_{c}^{\prime}, l^{\prime}\right)=\operatorname{Coder}\left(T^{c} \mathcal{H}_{o}\right)$ as above, its moduli space $\mathcal{M}\left(\mathcal{H}_{c}^{\prime}, \mathfrak{l}^{\prime}\right)$ is the moduli space of deformations of the $A_{\infty}$ algebra in the space of weak $A_{\infty}$ algebras. Thus, we have the following.

Corollary $6.4\left(A_{\infty}\right.$ structure parameterized by the moduli space of $L_{\infty}$ structures $\left.{ }^{35}\right)$ : For an $L_{\infty}$ algebra $\left(\mathcal{H}_{c}, \mathfrak{l}\right)$ and an $A_{\infty}$ algebra $\left(\mathcal{H}_{o}, \mathfrak{m}\right)$, suppose there exists an OCHA $\left(\mathcal{H}=\mathcal{H}_{c} \oplus \mathcal{H}_{o}, \mathfrak{l}, \mathfrak{n}\right)$ such that $\left(\mathcal{H}_{o},\left\{n_{0, k}\right\}\right)=\left(\mathcal{H}_{o}, \mathfrak{m}\right)$. Then, for each element $c \in \mathcal{M}\left(\mathcal{H}_{c}, \mathfrak{l}\right)$, we have a weak $A_{\infty}$ algebra which is a deformation of the original $A_{\infty}$ algebra $\left(\mathcal{H}_{o}, \mathfrak{m}\right)$. If $\left\{n_{1, k}\right\}_{k \geq 0}: \mathcal{H}_{c} \rightarrow \mathcal{H}_{c}^{\prime}$ gives a quasiisomorphism of complexes $\left(\mathcal{H}_{c}, l_{1}\right) \rightarrow\left(\mathcal{H}_{c}^{\prime}, l_{1}^{\prime}\right)$, then all the equivalence classes of deformations of $(\mathcal{H}, \mathfrak{m})$ as weak $A_{\infty}$ algebras, described by $\mathcal{M}\left(\mathcal{H}_{c}^{\prime}, l_{1}^{\prime}\right)$, are in one-to-one correspondence with the space $\mathcal{M}\left(\mathcal{H}_{c}, \mathfrak{l}\right)$.

\section{B. The construction of deformation quantization by Kontsevich}

The deformation quantization problem is to construct a star product corresponding to the Poisson algebra on a manifold $M$. Namely, for a formal (deformation) parameter $\nu$ and a given Poisson algebra $\left(\mathcal{A}=C^{\infty}(M), \cdot,\{\},\right)$, a bilinear, bidifferential $\nu$-linear map $*: \mathcal{A}[[\nu]] \otimes \mathcal{A}[[\nu]]$ $\rightarrow \mathcal{A}[[\nu]]$,

$$
f^{*} g=\sum_{r=0}^{\infty} m_{r}(f, g) \nu^{r}, \quad f, g \in \mathcal{A}[[\nu]]
$$

is called a deformation quantization of $M$ if $m_{0}(f, g)=f \cdot g$, the usual commutative product on $C^{\infty}(M), m_{1}(f, g)=\frac{1}{2}\{f, g\}$ and the star product $*$ is associative. ${ }^{5}$ (Notice here $m_{r}$ is still a function of two variables and should not be confused with the $m_{l}$ of an $A_{\infty}$ algebra.) In Ref. 40, Kontsevich reformulated this problem in a homotopy algebraic setup. For any associative algebra $\mathcal{A}$, deformations as associative multiplications are controlled by the Hochschild complex, which is essentially $\operatorname{Coder}\left(T^{c} \mathcal{A}\right)$ and hence a DGLA. ${ }^{12}$ In fact, control is equally well exercised by any quasiisomorphic DGLA or even $L_{\infty}$ algebra. ${ }^{60}$ The obstructions to existence and to equivalence are identified by the quasi-isomorphism. For the special case of $\mathcal{A}=C^{\infty}(M)$, the deformations relevant to deformation quantization are controlled by the subcomplex of multidifferential Hochschild cochains, which we denote $D_{\text {poly }}(M)$ (Definition 6.5) and which is quasi-isomorphic to the full Hochschild complex. The smooth analog of the Hochschild-Kostant-Rosenberg theorem. ${ }^{25}$ (an explicit proof can be found in Ref. 22) equates the Hochschild cohomology with $T_{\text {poly }}(M)$, the space of polyvector fields, which possesses a DGLA structures with $d=0$ and the SchoutenNijenhuis bracket.

Kontsevich treated these DGLAs as $L_{\infty}$ algebras and obtained the existence and the classification of deformation quantizations by constructing an $L_{\infty}$ morphism between $T_{\text {poly }}(M)$ and $D_{\text {poly }}(M)$, which is in a sense a nonlinear generalization of a DGLA map. Moreover, the specific $L_{\infty}$ morphism provides a specific star product. In this setting, the space of Poisson structures and the space of star products given by bidifferential operators are then described by the MaurerCartan equations of the corresponding DGLAs.

In this section, we shall first present these tools and then relate them to an OCHA.

Definition $6.5\left[D G L A\right.$ of multidifferential operators for $\left.C^{\infty}(M)\right]$ : For $\mathcal{A}=C^{\infty}(M)$, denote by $D_{\text {poly }}^{k}(M)$ the space of multilinear maps from $\mathcal{A}^{\otimes(k+1)}$ to $\mathcal{A}$ of multidifferential operators. Then define $D_{\text {poly }}=\oplus_{k \in \mathbb{Z}} D_{\text {poly }}^{k}$ by 


$$
D_{\text {poly }}^{k}=D_{\text {poly }}^{k}(M)(k \geqslant-1), \quad D_{\text {poly }}^{k}=0(k<-1) .
$$

For $d$, we take the Hochschild coboundary operator. Namely, for any $C \in D_{\text {poly }}^{k}, d$ is given by

$$
\begin{gathered}
(\mathrm{d} C)\left(g_{0}, \ldots, g_{k+1}\right)=g_{0} C\left(g_{1}, \ldots, g_{k+1}\right)-\sum_{r=0}^{k}(-1)^{r} C\left(g_{0}, \ldots, g_{r} g_{r+1}, \ldots, g_{k+1}\right) \\
+(-1)^{k} C\left(g_{0}, \ldots, g_{k}\right) g_{k+1} .
\end{gathered}
$$

We take for $[$,$] the Gerstenhaber bracket. { }^{12}$ Namely, for $C \in D_{\text {poly }}^{k}, C^{\prime} \in D_{\text {poly }}^{k^{\prime}}$, it is defined by

$$
\begin{gathered}
{\left[C, C^{\prime}\right]=C \circ C^{\prime}-(-1)^{k k^{\prime}} C^{\prime} \circ C,} \\
C \circ C^{\prime}\left(g_{0}, \ldots, g_{k+k^{\prime}}\right)=\sum_{r=0}^{k}(-1)^{r k^{\prime}} C\left(g_{0}, \ldots, g_{r-1}, C^{\prime}\left(g_{r}, \ldots, g_{r+k^{\prime}}\right), g_{r+k^{\prime}+1}, \ldots, g_{k+k^{\prime}}\right) .
\end{gathered}
$$

Then $\left(D_{\text {poly }},[], d,\right)$ forms a DGLA.

One can see that $d$ can be written as

$$
(-1)^{k}(d C)\left(g_{0}, \ldots, g_{k+1}\right)=[\bar{m}, C], \quad C \in D_{\text {poly }}^{k},
$$

where $\bar{m}$ is the usual commutative product of functions $\bar{m}(f, g)=f \cdot g$. Though the operation $[\bar{m}$, is different in sign from the original $d,[\bar{m}$,$] also forms a DGLA on D_{\text {poly }}$ with $[$,$] . So, we take$ this as $d$. This DGLA is described as a sub-DGLA of (Hom, $d$, [ , ]). First, set $\mathcal{H}_{o}^{-1}=\mathcal{A}$ and $\mathcal{H}_{o}^{k}$ $=0$ otherwise. Then $C: \mathcal{A}^{\otimes(k+1)} \rightarrow \mathcal{A}$ has degree $k$ as defined above. Namely, $D_{\text {poly }}(M)$ is included in the restricted subvector space $\mathrm{Hom}_{\mathrm{sub}}=\oplus_{k} \mathrm{Hom}_{k}^{k-1}$ of Hom.

Let us state the necessary condition for the existence of a deformation quantization in the language of DGLAs here. If we write the star product (6.3) as $f^{*} g=m(f, g), m \in \operatorname{Hom}_{2}^{1}$, the associativity condition $\left(f^{*} g\right) * h=f^{*}(g * h)$ is expressed algebraically as $[m, m]=0$. This just indicates that $m$ defines a codifferential on $T^{c}(\mathcal{A})$ as previously or, equivalently, that $m$ is associative. Since $m$ should be obtained as a deformation of $\bar{m}$, writing $m=\bar{m}+\theta, \theta \in \nu D_{\text {poly }}[[\nu]]$, one gets a Maurer-Cartan equation in the DGLA $\left(\nu D_{\text {poly }}[[\nu]], d,[],\right)$,

$$
\mathrm{d} \theta+\frac{1}{2}[\theta, \theta]=0 .
$$

Definition 6.6 (DGLA of polyvector fields): For $k \geqslant-1$, set $T_{\text {poly }}^{k}(M):=\Gamma\left(M, \wedge^{k+1} T M\right)$, and define $T_{\text {poly }}=\oplus_{k} T_{\text {poly }}^{k}$ by

$$
T_{\text {poly }}^{k}=T_{\text {poly }}^{k}(M)(k \geqslant-1), \quad T_{\text {poly }}^{k}=0(k<-1) .
$$

Here, when $k=-1$ we set $T_{\text {poly }}^{-1}=T_{\text {poly }}^{-1}(M)=C^{\infty}(M)$. The differential $d$ is defined by $d=0$. Therefore, the cohomology of the complex of $T_{\text {poly }}^{n}$ with respect to $d$ coincides with $T_{\text {poly }}^{n}$ itself. [ , ] is taken to be the Schouten-Nijenhuis bracket. ${ }^{61,56}$ For $\xi_{s}, \eta_{t} \in T_{\text {poly }}^{0}=\Gamma(M, T M)$, the bracket of $\xi_{0} \wedge \cdots \wedge \xi_{k}, \in T_{\text {poly }}^{k}$ with $\eta_{0} \wedge \cdots \wedge \eta_{l} \in T_{\text {poly }}^{l}, k, l \geqslant 0$, is defined by

$$
\begin{aligned}
& {\left[\xi_{0} \wedge \cdots \wedge \xi_{k}, \eta_{0} \wedge \cdots \wedge \eta_{l}\right]} \\
& \quad=\sum_{i=0}^{k} \sum_{j=0}^{l}(-1)^{s+t}\left[\xi_{s}, \eta_{t}\right] \wedge \xi_{0} \wedge \cdots \wedge \xi_{s-1} \wedge \xi_{s+1} \wedge \cdots \wedge \xi_{k} \wedge \eta_{0} \wedge \cdots \wedge \eta_{t-1} \wedge \eta_{t+1} \wedge \cdots \wedge \eta_{l},
\end{aligned}
$$

and for $k \geqslant 0, h \in T_{\text {poly }}^{-1}=C^{\infty}(M)$, the bracket is 


$$
\left[\xi_{0} \wedge \cdots \wedge \xi_{k}, h\right]=\sum_{r=0}^{k}(-1)^{r} \xi_{r}(h)\left(\xi_{0} \wedge \cdots \wedge \xi_{r-1} \wedge \xi_{r+1} \wedge \cdots \wedge \xi_{k}\right) .
$$

We can define $\mathcal{H}_{c}^{k-1}:=T_{\text {poly }}^{k}$ with this Schouten-Nijenhuis bracket.

Now, a bivector $\alpha=\sum_{i, j} \alpha^{i j}\left(\partial / \partial x_{i}\right) \wedge\left(\partial / \partial x_{j}\right) \in T_{\text {poly }}^{1}$ in the local expression represents the Poisson bracket by $\{f, g\}=\Sigma_{i, j} \alpha^{i j}\left(\partial f / \partial x_{i}\right)\left(\partial g / \partial x_{j}\right)$, where $\left(x_{1}, \ldots, x_{n}\right)$ are local coordinates of $M$. This bracket by definition satisfies all the axioms of a Poisson algebra except the Jacobi identity. The Jacobi identity is then described by

$$
[\alpha, \alpha]=0 .
$$

Since $d=0$, Eq. (6.5) is also the Maurer-Cartan equation. A bivector satisfying (6.5) is called a Poisson bivector. Similarly, a quantum deformation of the Poisson bivector is defined by $\alpha_{[\nu]}$ $\left.\in \nu T_{\text {poly }}^{1}[\nu]\right]$ satisfying

$$
\left[\alpha_{[\nu]}, \alpha_{[\nu]}\right]=0 .
$$

In the expansion $\alpha_{[\nu]}=\nu \alpha_{1}+\nu^{2} \alpha_{2}+\cdots$, the original classical Poisson bivector $\alpha$ is $\alpha_{1}$. In fact, expanding (6.6) in terms of powers of $\nu$, one can see that the lowest identity reads $\left[\alpha_{1}, \alpha_{1}\right]=0$.

Thus, the conditions that an element in $\nu T_{\text {poly }}^{1}[[\nu]]$ is a Poisson bracket and that an associative product on $C^{\infty}(M)[[\nu]]$ as an element in $\nu D_{\text {poly }}^{1}[[\nu]]$ are both described by Maurer-Cartan equations for DGLAs. Let us set two $L_{\infty}$ algebras $\left(\mathcal{H}_{c}, \mathfrak{l}\right)$ and $\left(\mathcal{H}_{c}^{\prime}, \mathfrak{l}^{\prime}\right)$ as the suspension of DGLAs $T_{\text {poly }}$ and $D_{\text {poly }}$, respectively. Namely, we have $\mathcal{H}_{c}^{k}=T_{\text {poly }}^{k+1}$ and $\mathcal{H}_{c}^{\prime k}=D_{\text {poly. }}^{k+1}$. The Maurer-Cartan equations for DGLAs (6.6), (6.4) are of course rewritten as the Maurer-Cartan equations for $L_{\infty}$ algebras through the suspension in Remark 6.2.

The existence and the classification of the deformation quantization follows from the formality theorem, ${ }^{40}$ which claims the existence of an $L_{\infty}$ quasi-isomorphism $\mathfrak{f}:\left(\mathcal{H}_{c}, \mathfrak{l}\right) \rightarrow\left(\mathcal{H}_{c}^{\prime}, \mathfrak{l}^{\prime}\right)$. Note that this fact implies that the DGLA of Hochschild cochains is (homotopically) formal; in particular, the higher $L_{\infty}$ structures vanish on its cohomologies. In order that $\mathfrak{f}$ is an $L_{\infty}$ quasiisomorphism, the chain map $f_{1}:\left(\mathcal{H}_{c}, d_{c}=0\right) \rightarrow\left(\mathcal{H}_{c}^{\prime}, d_{c}^{\prime}\right)$ must be a quasi-isomorphism, that is, $f_{1}$ must induce an isomorphism on cohomologies. One may set

$$
\left(f_{1}\left(\xi_{i_{1}} \wedge \cdots \wedge \xi_{i_{k}}\right)\left(g_{1}, \ldots, g_{k}\right)=\frac{1}{k !} \sum_{\sigma \in \mathfrak{S}}(-1)^{\epsilon(\sigma)}\left(\xi_{i_{\sigma(1)}} g_{1}\right) \cdots\left(\xi_{i_{\sigma(k)}} g_{k}\right)\right.
$$

Kontsevich constructs all the higher multilinear maps $f_{k}$ explicitly as local expressions on $M$ $=\mathbb{R}^{n}$ in terms of Feynman graphs, which are just those derived from a certain topological openclosed string theory as revealed explicitly by Cattaneo-Felder ${ }^{8}$ (see for review Ref. 48).

When an $L_{\infty}$ quasi-isomorphism $\mathfrak{f}=\left\{f_{1}, f_{2}, \ldots\right\}$ is given, it preserves the Maurer-Cartan equations, and the deformed Poisson bivector is given by the following nonlinear map:

$$
\theta=\sum_{k=1}^{\infty} \frac{1}{k !} f_{k}\left(\alpha_{[v]}, \ldots, \alpha_{[v]}\right),
$$

for $\alpha_{[\nu]} \in \mathcal{M}\left(\mathcal{H}_{c}, \mathfrak{l}\right)$ and $\theta \in \mathcal{M}\left(\mathcal{H}_{c}^{\prime}, \mathfrak{l}^{\prime}\right)$. Here the $L_{\infty}$ quasi-isomorphism $\mathfrak{f}:\left(\mathcal{H}_{c}, \mathfrak{l}\right) \rightarrow\left(\mathcal{H}_{c}^{\prime}, \mathfrak{l}^{\prime}\right)$ has been extended by tensoring with the formal power series $\nu \mathrm{C}[[\nu]]$. If we expand the deformation $m=\bar{m}+\theta, \theta \in \nu D_{\text {poly }}[[\nu]]$ as in Eq. (6.3):

$$
m=\bar{m}+\nu m_{1}+\nu^{2} m_{2}+\cdots
$$

with $m_{1}=(1 / 2) \alpha_{1}$, Kontsevich's quasi-isomorphism of $L_{\infty}$ algebras then provides a choice for $m_{2}$ and in fact for all the $m_{i}$.

Now, let us summarize Kontsevich's deformation quantization in terms of OCHAs. First, we set $\mathcal{H}_{c}^{r-2}:=\Gamma\left(M, \wedge^{r} T M\right)$. It forms a formal $L_{\infty}$ algebra with $l_{2}$ the Schouten-Nijenhuis bracket, and $l_{1}=l_{3}=\cdots=0$. For $\mathcal{H}_{o}$, we take $\mathcal{H}_{o}^{-1}:=C^{\infty}(M)$ and $\mathcal{H}_{o}^{k}=0$ for $k \neq-1$. The $A_{\infty}$ structure is $n_{0,2}=\bar{m}$, 
the usual commutative product of functions $C^{\infty}(M)$, and $n_{0, l}=0$ except for $l=2$. The multilinear maps of the $L_{\infty}$ quasi-isomorphism are then identified as the adjoints $\left\{n_{k, l}:\left(\mathcal{H}_{c}\right)^{\otimes k} \otimes\left(\mathcal{H}_{o}\right)^{\otimes l} \rightarrow \mathcal{H}_{o}\right\}$ with $k \geqslant 1$. In particular, $n_{1,2}\left(\alpha_{[\nu]} ; f, g\right)=(1 / 2)\{f, g\}, \quad$ and $\quad n_{1, k}\left(\xi_{i_{1}} \wedge \cdots \wedge \xi_{i_{k}} ; g_{1}, \ldots, g_{k}\right)$ $=(1 / k !) \Sigma_{\sigma \in \mathfrak{S}}(-1)^{\epsilon(\sigma)}\left(\xi_{i_{\sigma(1)}} g_{1}\right) \cdots\left(\xi_{\sigma_{\sigma(k)}} g_{k}\right)$. These structures form a minimal OCHA on cohomology. Corollary 6.4 then implies that, for a fixed element of $\mathcal{M C}\left(\mathcal{H}_{c}, \mathfrak{l}\right)$, an $A_{\infty}$ structure is obtained. However, in this situation, since Hom is restricted to $\mathrm{Hom}_{\mathrm{sub}}=\oplus \mathrm{Hom}_{r}^{r-1}$ and the elements of Maurer-Cartan equations, especially $\mathcal{M}\left(\mathcal{H}_{c}^{\prime}, \mathfrak{l}^{\prime}\right)$ are degree zero, the deformed $A_{\infty}$ structure also has $m_{2} \in\left(\operatorname{Hom}_{2}[1]\right)^{0}$ only, i.e., $m_{1}$ and higher product $m_{3}, m_{4}, \ldots$ are absent. Equivalently, when $\mathcal{H}_{c}$ is restricted to its degree zero part $\mathcal{H}_{c}^{0}, n_{k, l}$ vanishes except for $(k, l)=(k, 2)$. This $m_{2}$ is just the star product, an associative but noncommutative product $C^{\infty}(M) \otimes C^{\infty}(M) \rightarrow C^{\infty}(M)$ of a deformation quantization. The next example below is a natural extension of this situation, but in the case that Hom is not restricted to $\mathrm{Hom}_{\text {sub }}$.

\section{Open-closed B-model}

A natural extension of Kontsevich's deformation quantization setup is to the B-model side of homological mirror symmetry. ${ }^{39}$

The mirror symmetry, a symmetry between Calabi-Yau manifolds, can be interpreted as topological closed string physics. There are two types of topological string theories whose target spaces are Calabi-Yau manifolds. One is called the A-model, which depends only on the complexified symplectic structure and is independent of the complex structure of the Calabi-Yau manifold. Another one, the B-model in contrast depends only on the complex structure. For a given Calabi-Yau manifold $M$, the mirror symmetry conjecture is the existence of a mirror CalabiYau manifold $\hat{M}$ such that the A-model closed string on $\hat{M}$ is equivalent to the B-model closed string on $M$ and vice versa. ${ }^{71}$ Homological mirror symmetry is thought of as an open string version $^{72}$ of the mirror symmetry conjecture. open string theory in general includes some kind of D-branes, which form a D-brane category (see Ref. 45); the D-branes and open strings are identified with objects and morphisms between the objects. For the tree open string A-model, the corresponding category is Fukaya's $A_{\infty}$ category, ${ }^{9}$ which depends only on the complexified symplectic structure. On the other hand, what is constructed on the B-model side is a category of holomorphic vector bundles or coherent sheaves more generally. The homological mirror symmetry conjecture ${ }^{39}$ then states that the Fukaya category on a Calabi-Yau manifold $M$ is in some sense equivalent to the category of coherent sheaves on the mirror dual Calabi-Yau manifold $\hat{M}$. Now, the conjecture is checked successfully in the case $M$ is an elliptic curve, ${ }^{39,59,57}$ an Abelian variety ${ }^{10,41}$ a quartic surface, ${ }^{63}$ and so on. For noncommutative two-tori, see Refs. 32, 37, and 34 and a related work. ${ }^{58}$

One of the original motivations to argue for this homological mirror symmetry conjecture ${ }^{39}$ is that it might explain the (tree closed string parts of) mirror symmetry: the family (deformations) of tree open string A- (respectively, B-) models should be in one-to-one correspondence with that of tree closed string A-(respectively, B-) model, and the tree closed string structure should follow from the corresponding family of the tree open string structures. These concepts have their background in the open-closed string physics in our sense. Since the A side and B side should be mirror dual, they should have isomorphic structures in some sense. However, that which is directly related to us is the B-model side, since there the classical solution of string world sheet theory is only a constant map (no world sheet instanton) and the corresponding moduli spaces are just the usual ones of Riemann surfaces with boundaries and punctures. ${ }^{71}$

One can see that the tree open-closed B-model is just a particular example of the arguments in Sec. VI A, and gives a natural extension of that in the preceding section. However, the mathematical formulation of the tree open-closed B-model is not yet established completely, nor is there known the explicit formula as in the case of deformation quantization in the preceding section.

An interesting attempt and a partial result can be found in Hofman's work. ${ }^{26}$ We can identify some set of multilinear maps $\left\{l_{k}, n_{p, q}\right\}$ on open and closed string observables for this situation. Of course we could have an infinite number of homotopy equivalent open-closed homotopy struc- 
tures. For instance, for the tree closed string part, the world sheet action of the B-twisted topological string theory as given in Ref. 71 has the space of observables which is identified with the cohomology of $\oplus_{p, q} \Gamma\left(M, \wedge^{p} T M \otimes \wedge^{q} \bar{T}^{*} M\right)$ with respect to the Dolbeault operator $\bar{\partial}$ and hence, in principle, one can compute closed string $(k+1)$-point functions (=scattering amplitudes) related to $l_{k}$. However, in general this is complicated; it is better if one can find a corresponding string field theory in a simple form. Such a string field theory is given by Ref. 6, where the B-twisted topological closed string field theory action consists of the kinetic term and a three point vertex only, and so a DGLA structure is associated to it. Note that the equations of motions are just the Maurer-Cartan equations defining deformations of the complex structures, with additional extended directions mentioned below. The string field theory gives, at least for tree level (genus zero), a simple way of calculating $(k+1)$-point functions in terms of Feynman rules, and this procedure just coincides with taking a minimal model of the DGLA from a homotopy algebraic point of view. (However, for the action given in Ref. 6, they use an inner product which is not nondegenerate in our sense. In particular, it vanishes on the cohomology. Together with an additional structure called a differential Gerstenhaber structure, the pull-back of this action with respect to the $L_{\infty}$ quasi-isomorphism, or equivalently the superpotential or the collection of tree closed string amplitudes, has a Frobenius structure, ${ }^{6,2}$ even though the minimal $L_{\infty}$ structure is trivial, all $l_{k}=0$. We do not deal with this Frobenius structure in this paper.)

In a similar way, one can also consider open strings in the B-twisted topological string theory, and, in a similar spirit, one can construct a particular open string topological string field theory action, ${ }^{72,6}$ called a holomorphic Chern-Simons action, a holomorphic version of the usual ChernSimons action or Witten's bosonic open string field theory. ${ }^{70}$ Thus, it has a structure of a differential graded associative algebra (DGA) with cyclicity, a cyclic $A_{\infty}$ structure. Again, tree open string world sheet scattering amplitudes are obtained by taking the minimal model of the DGA.

Although the B-twisted topological string is controlled by such a simple DGLA or DGA for the closed or open string case, respectively, it is known that the same story does not hold for tree open-closed string in general. ${ }^{74}$ Therefore, we consider here a minimal OCHA structure whose purely closed string part $\left\{l_{k}\right\}$ and purely open string part $\left\{m_{k}=n_{0, k}\right\}$ are given by taking the minimal models of the DGLA and DGA, respectively. For the closed string side, the corresponding DGLA structure is $\left(\Gamma\left(M, \wedge^{\star} T M \otimes \wedge^{\star} \overline{T^{*} M}\right), \bar{\partial},[],\right)$, where [ ] is the Schouten-Nijenhuis bracket, the one in Definition 6.6 extended to $\overline{T^{*} M}$ naturally. However, for closed strings, the $\partial \bar{\partial}$ lemma and the Tian-Todorovs lemma lead to the corresponding minimal $L_{\infty}$ structure, given by the procedure in Sec. V, being trivial (see Refs. 6 and 2). Thus, we have $l_{k}=0$ fork $\geqslant 1$ for $\mathcal{H}_{c}^{k}=$ $\oplus_{p+q-2=k} H^{q}\left(\wedge^{p} T M\right)$, and the corresponding moduli space is itself,

$$
\mathcal{M}\left(\mathcal{H}_{c}, \mathfrak{l}\right)=\mathcal{H}_{c}^{0} .
$$

Here, we restrict $\mathcal{H}_{c}$ to its degree zero part only. It might be reasonable to think that one can deform in these $\mathcal{H}_{c}^{0}$ directions finitely in principle and that the other $\mathcal{H}_{c}^{k}$ directions provide fibers of an infinitesimal neighborhood. In fact, the whole deformation space $\mathcal{H}_{c}$ is called the extended moduli space in Barannikov-Kontsevich. ${ }^{2}$ Note that $H^{1}\left(\wedge^{1} T M\right) \in \mathcal{H}_{c}^{0}$ describes the complex structure deformations. This is the original deformation theory of complex structures, and the Barannikov-Kontsevich's setup can be thought of as an extension of it.

Next, for pure open string structure, we should stress what is taken for $\mathcal{H}_{o}$. The open string theory forms a D-brane category, which should be treated as an $A_{\infty}$ category in our context. The objects, B-type D-branes, are the coherent sheaves on $M$. Thus, $\mathcal{H}_{o}$ is identified with the space of morphisms between them. A general construction of a minimal model in this situation is found in Refs. 44, 46, 68, and 45, and See Ref. 34 for an explicit construction in the noncommutative two-tori case. However, for simplicity, here we consider the case that the object is only the structure sheaf $\mathcal{O}(M)$. One can see that this simplified situation is enough for our purpose here under some appropriate assumptions. The differential is then simply $\bar{\partial}$, and one obtains a minimal model of DGA $\left(\mathcal{O}^{0, *}(M), \bar{\partial}, \wedge\right)$, which we denote by $\left(\mathcal{H}_{o}, \bar{m}=\left\{n_{0, k}\right\}_{k \geqslant 2}\right)$, where $\mathcal{H}_{o}^{k-1}$ $=H^{k}\left(\mathcal{O}^{0, *}(M)\right)$. 
For this particular choice of $\mathcal{H}_{o}$, let us consider the space of multilinear maps Hom:= $\oplus \operatorname{Hom}_{k}^{r}$,

$$
\operatorname{Hom}_{k}^{r}=\operatorname{Hom}^{r}\left(\mathcal{H}_{o}^{\otimes k}, \mathcal{H}_{o}\right) .
$$

Again, the result of Gerstenhaber-Schack, ${ }^{13}$ in a similar way as the Hochschild-KonstantRosenberg theorem does in the preceding section, implies that the cohomology of Hom= $\oplus_{k, r} \operatorname{Hom}_{k}^{r}$ coincides with $\mathcal{H}_{c}$ itself (see Ref. 39).

Alternatively, the existence of an $L_{\infty}$ quasi-isomorphism from $\left(\mathcal{H}_{c}, \mathfrak{l}\right)$ to $\left(\mathcal{H}_{c}^{\prime}, \mathfrak{l}^{\prime}\right)$ is guaranteed at least physically, since $\left\{n_{k, l}\right\}$ can be constructed as open-closed disk amplitudes of the openclosed B-model, where the string world sheet action is of the same form as the one for pure closed strings, and the space of observables are just those used in separate open-closed B-model. An open-closed disk amplitude is then obtained by the integral of a disk correlation function, calculated in the usual way in physics, over the moduli space of the corresponding disk with punctures, both in the interior and on the boundary.

Moreover, it was checked in Ref. 26 that $n_{1, q}$ given by this physical argument in fact gives the linear part of the $L_{\infty}$ quasi-isomorphism $f_{1}$,

$$
n_{1, k}\left(\xi_{i_{1}} \wedge \cdots \wedge \xi_{i_{k}}, f_{1}, \ldots, f_{k}\right)=\frac{1}{k !} \sum_{\sigma \in \mathfrak{S}}(-1)^{\epsilon(\sigma)}\left(\xi_{i_{\sigma(1)}} f_{1}\right) \cdots\left(\xi_{i_{\sigma(k)}} f_{k}\right),
$$

where $\xi_{i} \in T M$ acts on $f_{i} \in \mathcal{H}_{o}$ as the Lie derivative. Also, in the spirit of homological mirror symmetry, since the collection $\left\{n_{k, l}\right\}$ gives an $L_{\infty}$ quasi-isomorphism even with the restriction to the category of coherent sheaves to $\mathcal{O}(M)$, it does also in case the full category would be treated for the open string side.

To summarize, we set $\mathcal{H}_{c}^{k}=\oplus_{p+q-2=k} H^{q}\left(\wedge^{p} T M\right), \quad \mathcal{H}_{o}^{k-1}=H^{k}\left(\mathcal{O}^{0, *}(M)\right), \quad$ and $\quad \operatorname{Hom}_{k}^{r}$ $=\operatorname{Hom}^{r}\left(\mathcal{H}_{o}^{\otimes k}, \mathcal{H}_{o}\right)$. Then we have $l_{k}=0$ on $\mathcal{H}_{c}$ and $n_{0, q}$ is the minimal $A_{\infty}$ structure of DGA $\left(\mathcal{O}^{0, *}(M), \bar{\partial}, \wedge\right)$. The operation $l^{\prime}$ is defined as in Sec. VI A, that is, $l_{1}^{\prime}=[\bar{m}$,$] with \bar{m}=\Sigma_{0, q} n_{0, q}$ and $\mathfrak{r}_{2}^{\prime}$ is related to the commutator $[$,$] through the suspension.$

The corresponding OCHA structure in this case reduces to the generalized WDVV relation discussed in Ref. 24. Namely, it corresponds to a minimal OCHA with trivial $L_{\infty}$ structure. At present, the explicit form of multilinear maps $n_{p, q}$ of $p \geqslant 2$ and $q \geqslant 0$ are not known in general, though physically existence is guaranteed by the open-closed scattering amplitudes of disks in B-twisted topological string theory. There is an interesting restriction where we can understand the form of $n_{k, l}$. Hofman discussed in Ref. 26 that if we restrict $\mathcal{H}_{c}$ to $\wedge^{p} T M$, the situation reduces to a complex version of Kontsevich's deformation quantization and hence $n_{p, q}$ are obtained in a similar way.

An interesting difference from the deformation quantization setup in the preceding section is that, since $\mathrm{Hom}=\oplus \operatorname{Hom}_{k}^{r}$ is not restricted to $\mathrm{Hom}_{\text {sub }}$, even if $r=1$ we can have nontrivial deformed $m_{k}$. In particular, we could in general obtain a deformation of the $A_{\infty}$ structure $\bar{m}$ to a weak $A_{\infty}$ structure, which should be one of the interesting future directions to be investigated.

In this case, for the closed string part, the $L_{\infty}$ structure is trivial, including the bracket, and hence the obstructions vanish. In a more general model, however, it should not be trivial, as discussed by Huebschmann-Stasheff. ${ }^{29}$ It should be interesting to find such models which can be calculated explicitly.

\section{ACKNOWLEDGMENTS}

One of the authors (H.K.) is supported by JSPS Research Fellowships for Young Scientists. One of the authors (J.S.) is supported in part by NSF Grant No. FRG DMS-0139799 and USCzech Republic Grant No. INT-0203119.The authors would like to thank T. Kimura, E. Harrelson, M. Markl, and A. Voronov for valuable discussions. 
${ }^{1}$ Alexandrov, M., Kontsevich, M., Schwartz, A., and Zaboronsky, O., "The geometry of the master equation and topological quantum field theory,” Int. J. Mod. Phys. A 12, 1405 (1997).

${ }^{2}$ Barannikov, S. and Kontsevich, M., "Frobenius manifolds and formality of Lie algebras of polyvector fields," Int. Math. Res. Notices 1998, 201-215 (1998).

${ }^{3}$ Batalin, I. A. and Vilkovisky, G. A., "Gauge algebra and quantization," Phys. Lett. B 102, 27 (1981).

${ }^{4}$ Batalin, I. A. and Vilkovisky, G. A., "Quantization of gauge theories with linearly dependent generators," Phys. Rev. D 28, 2567 (1983); 30, 508(E) (1983).

${ }^{5}$ Bayen, F., Flato, M., Frønsdal, C., Lichnerowicz, A., and Sternheimer, D., "Deformation theory and quantization I; II," Ann. Phys. 111, 61-110 (1978); 111, 111-151 (1978).

${ }^{6}$ Bershadsky, M., Cecotti, S., Ooguri, H., and Vafa, C., "Kodaira-Spencer theory of gravity and exact results for quantum string amplitudes," Commun. Math. Phys. 165, 311-427 (1994).

${ }^{7}$ Boardman, J. M. and Vogt, R. M., Homotopy Invariant Algebraic Structures on Topological Spaces, Lecture Notes in Mathematics 347 (Springer, New York, 1973).

${ }^{8}$ Cattaneo, A. S. and Felder, G., "A path integral approach to the Kontsevich quantization formula," Commun. Math. Phys. 212, 591-611 (2000).

${ }^{9}$ Fukaya, K., "Morse homotopy, $A^{\infty}$-category, and Floer homologies," Proceedings of GARC Workshop on Geometry and Topology '93 (Seoul, 1993), pp. 1-102, Lecture Notes Ser., 18, Seoul Nat. Univ., Seoul, 1993.

${ }^{10}$ Fukaya, K., "Mirror symmetry of abelian varieties and multi theta functions," J. Algeb. Geom. 11, 393-512 (2002).

${ }^{11}$ Gaberdiel, M. R. and Zwiebach, B., "Tensor constructions of open string theories I: Foundations," Nucl. Phys. B 505, 569 (1997).

${ }^{12}$ Gerstenhaber, M., "The cohomology structure of an associative ring," Ann. Math. 78, 267-288 (1963); "On the deformation of rings and algebras," ibid. 79, 59-103 (1964).

${ }^{13}$ Gerstenhaber, M. and Schack, S. D., "Algebraic cohomology and deformation theory," Deformation Theory of Algebras and Structures and Applications (Il Ciocco, 1986), NATO Adv. Sci. Inst. Ser. C Math. Phys. Sci., 247 (Kluwer Academic, Dordrecht, 1988), pp. 11-264.

${ }^{14}$ Getzler, E., "Batalin-Vilkovisky algebras and two-dimensional topological field theories," Commun. Math. Phys. 159, 265 (1994).

${ }^{15}$ Getzler, E. and Jones, J. D. S., "A $A_{\infty}$-algebras and the cyclic bar complex," Ill. J. Math. 34, 256 (1990).

${ }^{16}$ Getzler, E. and Kapranov, M. M., "Cyclic operads and cyclic homology," Geometry, Topology, and Physics, Conf. Proc. Lecture Notes Geom. Topology, IV (International Press, Cambridge, MA, 1995), pp. 167-201.

${ }^{17}$ Gugenheim, V. K. A. M., "On a perturbation theory for the homology of the loop-space," J. Pure Appl. Algebra 25, 197-205 (1982).

${ }^{18}$ Gugenheim, V. K. A. M. and Lambe, L. A., "Perturbation theory in differential homological algebra. I," Ill. J. Math. 33, 566-582 (1989).

${ }^{19}$ Gugenheim, V. K. A. M., Lambe, L. A., and Stasheff, J. D., “Algebraic aspects of Chen's twisting cochain,” Ill. J. Math. 34, 485-502 (1990).

${ }^{20}$ Gugenheim, V. K. A. M., Lambe, L. A., and Stasheff, J. D., "Perturbation theory in differential homological algebra II," Ill. J. Math. 35, 357-373 (1991).

${ }^{21}$ Gugenheim, V. K. A. M. and Stasheff, J. D., "On perturbations and $A_{\infty}$-structures," Bull. Soc. Math. Belg., Ser. A 38, 237-246 (1987).

${ }^{22}$ Gutt, S. and Rawnsley, J., "Equivalence of star products on a symplectic manifold; an introduction to Deligne's Cech cohomology classes," J. Geom. Phys. 29, 347-392 (1999).

${ }^{23}$ Harrelson, E., "On the homology of open/closed string theory," math.AT/0412249.

${ }^{24}$ Herbst, M., Lazaroiu, C. I., and Lerche, W., "Superpotentials, A(infinity) relations and WDVV equations for open topological strings," J. High Energy Phys. 0502, 071 (2005).

${ }^{25}$ Hochschild, G., Kostant, B., and Rosenberg, A., "Differential forms on regular affine algebras," Trans. Am. Math. Soc. 102, 383-408 (1962)

${ }^{26}$ Hofman, C., “On the open-closed B-model,” J. High Energy Phys. 0311, 069 (2003).

${ }^{27}$ Hofman, C. and Ma, W. K., "Deformations of topological open strings," J. High Energy Phys. 0101, 035 (2001).

${ }^{28}$ Huebschmann, J. and Kadeishvili, T., "Small models for chain algebras," Math. Z. 207, 245-280 (1991).

${ }^{29}$ Huebschmann, J. and Stasheff, J., "Formal solution of the master equation via HPT and deformation theory," Forum Math. 14, 847-868 (2002).

${ }^{30}$ Kadeishvili, T. V., "The algebraic structure in the homology of an $A(\infty)$-algebra," Soobshch. Akad. Nauk Gruz. SSR 108, 249-252 (1983).

${ }^{31}$ Kajiura, H., "Homotopy algebra morphism and geometry of classical string field theories," Nucl. Phys. B 630, 361 (2002).

${ }^{32}$ Kajiura, H., "Kronecker foliation, D1-branes and Morita equivalence of noncommutative two-tori," J. High Energy Phys. 0208, 050 (2002).

${ }^{33}$ Kajiura, H., "Noncommutative homotopy algebras associated with open strings," thesis submitted to Graduate School of Mathematical Sciences, University of Tokyo, math.QA/0306332.

${ }^{34}$ Kajiura, H., "Homological mirror symmetry on noncommutative two-tori," hep-th/0406233.

${ }^{35}$ Kajiura, H. and Stasheff, J., "Homotopy algebras inspired by classical open-closed string field theory," math.QA/ 0410291 .

${ }^{36}$ Kajiura, H. and Terashima, Y., "Homotopy equivalence of $A_{\infty}$-morphisms and gauge transformations," 2003.

${ }^{37} \mathrm{Kim}$, E. and Kim, H., "Moduli spaces of standard holomorphic bundles on a noncommutative complex torus," math.QA/ 0312228 .

${ }^{38}$ Kimura, T., Stasheff, J., and Voronov, A. A., "On operad structures of moduli spaces and string theory," Commun. Math. 
Phys. 171, 1 (1995).

${ }^{39}$ Kontsevich, M., "Homological algebra of mirror symmetry," Proceedings of the International Congress of Mathematicians (Zürich, 1994) (Birkhäuser, Basel, 1995), Vols. 1, and 2, pp. 120-139.

${ }^{40}$ Kontsevich, M., "Deformation quantization of Poisson manifolds," Lett. Math. Phys. 66, 157-216 (2003).

${ }^{41}$ Kontsevich, M. and Soibelman, Y., "Homological mirror symmetry and torus fibrations," Symplectic geometry and mirror symmetry (Seoul, 2000), 203-263 (World Sci. Publishing, River Edge, NJ), 2001, math.SG/0011041.

${ }^{42}$ Lada, T. and Markl, M., "Strongly homotopy Lie algebras," Commun. Algebra 23, 2147-2161 (1995).

${ }^{43}$ Lada, T. and Stasheff, J., "Introduction to sh Lie algebras for physicists," Int. J. Theor. Phys. 32, 1087-1103 (1993).

${ }^{44}$ Lazaroiu, C. I., "String field theory and brane superpotentials," J. High Energy Phys. 0110, 018 (2001).

${ }^{45}$ Lazaroiu, C. I., "D-brane categories,” Int. J. Mod. Phys. A 18, 5299 (2003).

${ }^{46}$ Lazaroiu, C. I. and Roiban, R., "Holomorphic potentials for graded D-branes," J. High Energy Phys. 0202, 038 (2002); Lazaroiu, C. I. and Roiban, R., "Gauge-fixing, semiclassical approximation and potentials for graded Chern-Simons theories," ibid. 0203, 022 (2002).

${ }^{47}$ Lefèvre-Hasegawa, K., "Sur les $A_{\infty}$-catégories," math.CT/0310337.

${ }^{48}$ Maeda, Y. and Kajiura, H., "String theory and deformation quantization," Sugaku Expo. 55, 245-265 (2003).

${ }^{49}$ Markl, M., "Models for operads," Commun. Algebra 24, 1471-1500 (1996).

${ }^{50}$ Markl, M., "Loop homotopy algebras in closed string field theory," Commun. Math. Phys. 221, 367 (2001)

${ }^{51}$ Mark1, M., "Homotopy algebras are homotopy algebras," Forum Math. 16, 129 (2004), math.AT/9907138.

${ }^{52}$ Markl, M., "Transferring $A_{\infty}$ (strongly homotopy associative) structures," math.AT/0401007.

${ }^{53}$ Markl, M., Shnider, S., and Stasheff, J., Operads in Algebra, Topology and Physics, Mathematical Surveys and Monographs, 96 (American Mathematical Society, Providence, RI, 2002).

${ }^{54}$ Merkulov, S. A., "Strong homotopy algebras of a Kähler manifold," Int. Math. Res. Notices 1999, 153-164 (1999).

${ }^{55}$ Nakatsu, T., "Classical open string field theory: A(infinity)-algebra, renormalization group and boundary states," Nucl. Phys. B 642, 13 (2002).

${ }^{56}$ Nijenhuis, A., "Jacobi-type identities for bilinear differential concomitants of certain tensor fields I; II," Ind. Math. 17, 390-397 (1955); 17, 398-403 (1955).

${ }^{57}$ Polishchuk, A., "Homological mirror symmetry with higher products," Winter School on Mirror Symmetry, Vector Bundles and Lagrangian Submanifolds (Cambridge, MA, 1999), 247-259, AMS/IP Stud. Adv. Math., 23 (Amer. Math. Soc., Providence, RI 2001), math.AG/9901025; "A $A_{\infty}$-structures on an elliptic curve," Commun. Math. Phys. 247, 527 (2004), math.AG/0001048.

${ }^{58}$ Polishchuk, A. and Schwarz, A., "Categories of holomorphic vector bundles on noncommutative two-tori," Commun. Math. Phys. 236, 135 (2003).

${ }^{59}$ Polishchuk, A. and Zaslow, E., "Categorical mirror symmetry: the elliptic curve," Adv. Theor. Math. Phys. 2, 443-470 (1998).

${ }^{60}$ Schlessinger, M. and Stasheff, J., "Deformaion theory and rational homotopy type," University of North Carolina, 1979; "The Lie algebra structure of tangent cohomology and deformation theory," J. Pure Appl. Algebra 38, 313-322 (1985).

${ }^{61}$ Schouten, J. A., "On the differential operators of first order in tensor calculus," Convegno Internazionale di Geometria Differenziale, Italia (Edizioni Cremonese, Roma, 1954, pp. 1-7).

${ }^{62}$ Schwarz, A., "Geometry of Batalin-Vilkovisky quantization," Commun. Math. Phys. 155, 249 (1993).

${ }^{63}$ Seidel, P., "Homological mirror symmetry for the quartic surface," math.SG/0310414.

${ }^{64}$ Stasheff, J. D., "On the homotopy associativity of $H$-spaces, I; II," Trans. Am. Math. Soc. 108, 275 (1963).

${ }^{65}$ Stasheff, J. D., "The intrinsic bracket on the deformation complex of an associative algebra," J. Pure Appl. Algebra 89, 231-235 (1993).

${ }^{66}$ Stasheff, J., "Closed string field theory, strong homotopy Lie algebras and the operad actions of moduli spaces," hep-th/9304061.

${ }^{67}$ Stasheff, J., "From operads to "physically" inspired theories," Operads: Proceedings of Renaissance Conferences (Hartford, CT/Luminy, 1995), Contemp. Math. 202 (American Mathematical Society, Providence, RI, 1997), pp. 53-81.

${ }^{68}$ Tomasiello, A., "A-infinity structure and superpotentials," J. High Energy Phys. 0109, 030 (2001).

${ }^{69}$ Voronov, A., "The Swiss-cheese operad," Homotopy Invariant Algebraic Structures (Baltimore, MD, 1998), Contemp. Math. 239 (American Mathematical Society, Providence, RI, 1999), pp. 365-373.

${ }^{70}$ Witten, E., "Noncommutative geometry and string field theory," Nucl. Phys. B 268, 253 (1986).

${ }^{71}$ Witten, E., "Mirror manifolds and topological field theory," Essays on Mirror manifolds (International Press, Hong Kong, 1992), pp. 120-158.

${ }^{72}$ Witten, E., "Chern-Simons gauge theory as a string theory," Prog. Math. 133, 637 (1995).

${ }^{73}$ Zwiebach, B., "Closed string field theory: Quantum action and the B-V master equation," Nucl. Phys. B 390, 33 (1993).

${ }^{74}$ Zwiebach, B., "Oriented open-closed string theory revisited," Annals Phys. 267, 193 (1998), hep-th/9705241. 